\title{
The EPOCH Project
}

\section{Periodic variable stars in the EROS-2 LMC database ${ }^{\star}$}

\author{
Dae-Won Kim ${ }^{1}$, Pavlos Protopapas ${ }^{2}$, Coryn A. L. Bailer-Jones ${ }^{1}$, Yong-Ik Byun ${ }^{3}$, Seo-Won Chang ${ }^{3}$, \\ Jean-Baptiste Marquette ${ }^{4}$, and Min-Su Shin ${ }^{5}$
}

\author{
1 Max-Planck Institute for Astronomy, Königstuhl 17, 69117 Heidelberg, Germany \\ e-mail: kim@mpia.de \\ 2 Harvard-Smithsonian Center for Astrophysics, 60 Garden, Cambridge MA 02138, USA \\ 3 Department of Astronomy and University Observatory, Yonsei University, 50 Yonsei-Ro, 120-749 Seoul, South Korea \\ ${ }^{4}$ UPMC-CNRS, UMR7095, Institut d'Astrophysique de Paris, 75014 Paris, France \\ 5 Department of Physics, Oxford University, Parks Road, Oxford OX1 3PU, UK
}

Received 13 December 2013 / Accepted 24 March 2014

\section{ABSTRACT}

\begin{abstract}
The EPOCH (EROS-2 periodic variable star classification using machine learning) project aims to detect periodic variable stars in the EROS-2 light curve database. In this paper, we present the first result of the classification of periodic variable stars in the EROS-2 LMC database. To classify these variables, we first built a training set by compiling known variables in the Large Magellanic Cloud area from the OGLE and MACHO surveys. We crossmatched these variables with the EROS-2 sources and extracted 22 variability features from 28392 light curves of the corresponding EROS-2 sources. We then used the random forest method to classify the EROS-2 sources in the training set. We designed the model to separate not only $\delta$ Scuti stars, RR Lyraes, Cepheids, eclipsing binaries, and long-period variables, the superclasses, but also their subclasses, such as RRab, RRc, RRd, and RRe for RR Lyraes, and similarly for the other variable types. The model trained using only the superclasses shows $99 \%$ recall and precision, while the model trained on all subclasses shows $87 \%$ recall and precision. We applied the trained model to the entire EROS-2 LMC database, which contains about 29 million sources, and found 117234 periodic variable candidates. Out of these 117234 periodic variables, 55285 have not been discovered by either OGLE or MACHO variability studies. This set comprises $1906 \delta$ Scuti stars, 6607 RR Lyraes, 638 Cepheids, 178 Type II Cepheids, 34562 eclipsing binaries, and 11394 long-period variables.
\end{abstract}

Key words. stars: variables: general - Magellanic Clouds - methods: data analysis

\section{Introduction}

Studying periodic variable stars has improved our understanding of the Universe for many decades. For instance, Cepheid variables are one of the most important variable types as a standard candle for measuring extra-galactic distances (Freedman et al. 2001; Riess et al. 2011) because of their well-established periodluminosity relation (Feast \& Catchpole 1997; Storm et al. 2011), which provided evidence for the expanding Universe (Lemaitre 1927; Hubble \& Humason 1931). RR Lyrae stars are useful for tracing the Galaxy formation history (e.g. see Catelan 2009, and references therein) and for studying globular clusters (Carretta et al. 2000) and the Galactic structure (Oort \& Plaut 1975; Vivas et al. 2001). In addition, long-period variables such as Mira variables show a period-luminosity relation that can be used for measuring distances to some objects in the Galaxy, such as globular clusters (Feast et al. 1989; Knapp et al. 2003). In brief, periodic variable stars are crucial for studying and understanding the Galaxy and the Universe.

The Expérience pour la Recherche d'Objets Sombres (EROS) is a wide-field sky survey for probing dark matter in the Galactic halo by detecting microlensing events (see Tisserand et al. 2007, and references therein). EROS monitored

* A catalog of these EROS-2 LMC periodic variable stars is available at http://stardb.yonsei.ac. $\mathrm{kr}$ and at the CDS via anonymous ftp to cdsarc.u-strasbg. fr (130.79.128.5) or via http://cdsarc.u-strasbg.fr/viz-bin/qcat?]/A+A/566/A43 the Large/Small Magellanic Cloud (LMC/SMC), the Galactic bulge and spiral arms for about seven years, and was a major microlensing survey together with the MACHO (Alcock 2000) and OGLE (Udalski et al. 1997) microlensing surveys. In addition to microlensing detections, the EROS database is also useful for detecting periodic variable stars because of its well-sampled light curves over a long period of observation, relatively faint limiting magnitude of $\sim 20$ in the EROS $B$ band $^{1}$, its wide field of view, and two simultaneous passbands.

Previous studies have found several types of variable stars in the EROS-2 database. Beaulieu et al. (2001) discovered two variable stars resembling Herbig Ae/Be or classical Be stars, Tisserand et al. (2004) detected five R Coronae Borealis stars, Marquette et al. (2009) discovered 185 new beat Cepheid variables, Spano et al. (2011) reported forty-three thousand longperiod variable candidates, and Dubath et al. (2012) found about 300 variable candidates from a subset of EROS-2 database but without identifying their variable types. Although these works produced some variable star candidates of various types, no study has searched the entire EROS-2 light curve database to classify different types of variable stars including $\delta$ Scuti stars, RR Lyraes, Cepheids, eclipsing binaries, and long-period variables. For instance, Dubath et al. (2012) used a supervised machine-learning method and multiple variability features to train a classification model, but their training set is incomplete

1 EROS $B$ and $R$ bands (i.e., $B_{\mathrm{E}}$ and $R_{\mathrm{E}}$ ) are not standard astronomical $B$ and $R$ bands. See Sect. 2 for details. 
because it consists of visually classified variables sorted into four classes including periodic, small-amplitude, semi-regular, and nonperiodic variables. Thus the model cannot distinguish conventional types of astronomical variable stars such as the types of variable stars listed above.

We initiated the EROS-2 PeriOdic variable star Classification using macHine learning (EPOCH) project ${ }^{2}$ that aims to detect periodic variables in the EROS-2 light curve database to significantly increase the total number of known variable stars in the EROS-2 survey fields. The EPOCH project is different from the previous studies because we used 1) the richest possible training set including multiple types of variable stars; 2) a few tens of variability features to separate variable stars from others; and 3 ) one of the most powerful supervised classification methods, random forest (Breiman 2001). Random forest combines a large number of decision trees to build a classification model and has successfully solved many astronomical classification problems (e.g. Dubath et al. 2011; Pichara et al. 2012). Even though some of the above conditions were fulfilled by previous work, none of the works has fulfilled all of these conditions simultaneously.

In this paper, we present the first results of the EPOCH project. We also present periodic variable star candidates detected from the EROS-2 LMC light curve database. The EROS-2 database is briefly introduced in Sect. 2. Section 3 presents a classification method including 1) the training set we used to build a classification model; 2) multiple variability features; 3 ) parameter optimization for the random forest model training; and 4) performance of a trained model. We then show detection results of periodic variable stars from the entire EROS-2 LMC database in Sect. 4. Section 5 is a summary.

\section{EROS-2 database}

EROS-2 monitored the LMC/SMC, the Galactic center, and the spiral arms during its operation from July 1996 to March 2003 using the $1 \mathrm{~m}$ Ritchey-Chrétien Telescope, MARLY, at ESO (La Silla, Chile). The telescope was equipped with two cameras, one observing in the $B_{\mathrm{E}}(420-720 \mathrm{~nm}$, blue) band, the other in the $R_{\mathrm{E}}(620-920 \mathrm{~nm}$, red) band. Each camera contained eight $2048 \times 2048$ CCD detectors in mosaics, and had a field of view of $0.7^{\circ} \times 1.4^{\circ}$ in RA and Dec, respectively. The pixel scale was $0.6^{\prime \prime}$ and the typical seeing at the site was $2^{\prime \prime}$ FWHM (Ansari \& EROS Collaboration 2001; Tisserand et al. 2004).

The total number of sources in the EROS-2 database is about 87 million consisting of 29 million in the LMC, 4 million in the SMC, 44 million in the Galactic bulge, and 10 million sources in the spiral arms. Light curves obtained using the $B_{\mathrm{E}}$ band generally have better photometric accuracy and also have more measurements than $R_{\mathrm{E}}$-band light curves (Spano et al. 2011). Figure 1 shows a histogram of the $B_{\mathrm{E}}$ band magnitude of about $550 \mathrm{k}$ sources in the EROS-2 LMC fields. The limiting magnitude of the field sources is $\sim 20 B_{\mathrm{E}}$. The number of measurements for each light curve varies from field to field. For instance, $B_{\mathrm{E}}$-band light curves around the central region of the LMC have 500 measurements on average, while $B_{\mathrm{E}}$-band light curves at the outer region have 250 measurements on average.

\section{Classification of periodic variables}

In this section, we describe how we classify periodic variable stars in the EROS-2 LMC light-curve database. In Sect. 3.1, we

\footnotetext{
2 http://stardb.yonsei .ac.kr
}

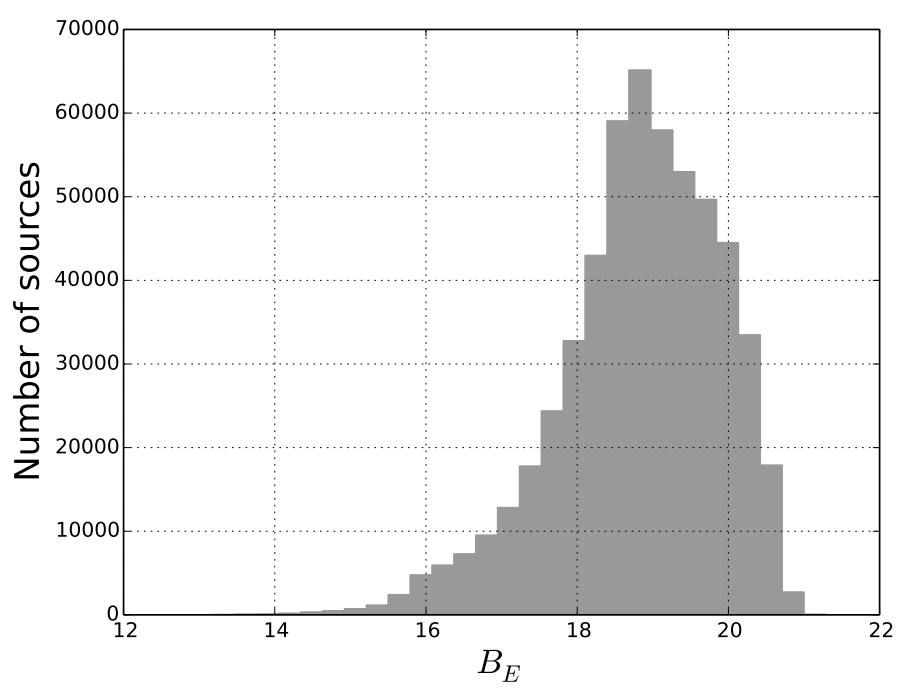

Fig. 1. Histogram of EROS $B_{\mathrm{E}}$ band magnitude of about 550k field sources from the EROS-2 LMC fields. The limiting magnitude is around $20 B_{\mathrm{E}}$.

Table 1. Acronyms of each variable type.

\begin{tabular}{cc}
\hline \hline Variable type & Acronym \\
\hline$\delta$ Scuti & DSCT \\
RR Lyrae & RRL \\
Cepheid & CEPH \\
Type II Cepheid & T2CEPH \\
Eclipsing binary & EB \\
Long-period variable & LPV \\
Blue variable & BV \\
\hline
\end{tabular}

present a training set consisting of EROS-2 variable stars selected by crossmatching with the known OGLE and MACHO variables. Section 3.2 explains how we refined the light curve (e.g., cleaning spurious data points) before we extracted 22 variability features that are described in Sect. 3.3. We then present the random forest classification algorithm and summarize its performance in Sect. 3.4.

\subsection{Training set}

The classification quality of supervised machine-learning methods relies heavily on the training set. To build the richest possible training set, we therefore used the OGLE LMC variable star catalogs (Soszyński et al. 2008a,b, 2009a,b; Poleski et al. 2010; Graczyk et al. 2011), which are the most up-to-date and complete catalogs of periodic variable stars over the EROS-2 fields. Based on these catalogs, we compiled a list of periodic variable stars including $\delta$ Scuti stars, RR Lyraes, Cepheids, Type II Cepheids, eclipsing binaries, and long-period variables (DSCTs, RRLs, CEPHs, T2CEPHs, EBs and LPVs, respectively, Table 1). We adopted the OGLE classification scheme for variable subclasses (e.g. RRL ab, c, d, and e types; see Table 2 for the full list). We added 982 blue variables detected from the MACHO database (Keller et al. 2002). Many of these blue variables are probably Be stars since their variability characteristics are consistent with those of Be stars (Keller et al. 2002). We also added 565 quasi-stellar objects (QSOs) to the list that were selected using the OGLE LMC database (Kozlowski et al. 2013). Although we do not aim to classify either blue variables (BVs) or QSOs 
D.-W. Kim et al.: The EPOCH Project. I.

Table 2. Number of remaining variables after crossmatching, visual removal, and light-curve refining.

\begin{tabular}{|c|c|c|c|c|c|}
\hline Superclass & Subclass & $\begin{array}{l}\text { After crossmatching } \\
\text { (number) }\end{array}$ & $\begin{array}{l}\text { After visual removal } \\
\quad\left(\text { number }\left(\%^{a}\right)\right)\end{array}$ & $\begin{array}{l}\text { After refining } \\
\left(\text { number }\left(\%^{a}\right)\right)\end{array}$ & Note \\
\hline DSCT & & 1155 & $511(44)$ & $511(44)$ & \\
\hline \multicolumn{6}{|l|}{ CEPH } \\
\hline & fundamental $(\mathrm{F})$ & 1275 & $1275(100)$ & $1249(98)$ & \\
\hline & first overtone (1O) & 871 & $844(97)$ & $834(96)$ & \\
\hline & Other & 168 & $162(96)$ & $160(95)$ & \\
\hline \multicolumn{6}{|l|}{ RRL } \\
\hline & $a b$ & 8721 & 3434 (39) & $3276(38)$ & \\
\hline & $\mathrm{c}$ & 2461 & $714(29)$ & $637(26)$ & \\
\hline & d & 511 & $179(35)$ & $172(34)$ & \\
\hline & $\mathrm{e}$ & 603 & $143(24)$ & $141(23)$ & \\
\hline \multicolumn{6}{|l|}{ EB } \\
\hline & $\mathrm{EC}$ & 605 & 445 (74) & 418 (69) & contact \\
\hline & ED & 8615 & 1644 (19) & 1592 (19) & detached \\
\hline & ESD & 3409 & $1150(34)$ & $1109(33)$ & semi-detached \\
\hline & $\mathrm{ED}+\mathrm{ESD}$ & 870 & $146(17)$ & $142(16)$ & detached/semi-detached \\
\hline & Other & 254 & $153(60)$ & $146(58)$ & \\
\hline LFv & Mira AGB C & 785 & $754(96)$ & $567(72)$ & carbon-rich \\
\hline & Mira AGB O & 329 & $329(100)$ & $324(99)$ & oxygen-rich \\
\hline & OSARG AGB C & 2119 & $1416(67)$ & $1135(54)$ & carbon-rich \\
\hline & OSARG AGB O & 22670 & $3368(15)$ & $3187(14)$ & oxygen-rich \\
\hline & OSARG RGB C & 264 & $57(22)$ & $46(17)$ & carbon-rich \\
\hline & OSARG RGB O & 32648 & $2046(6)$ & $1888(6)$ & oxygen-rich \\
\hline & SRV AGB C & 3889 & $3819(98)$ & $2507(65)$ & carbon-rich \\
\hline & SRV AGB O & 4482 & $4206(94)$ & $3672(82)$ & oxygen-rich \\
\hline T2CEPH & & 123 & $121(98)$ & $112(91)$ & \\
\hline BV & & 829 & $796(96)$ & 725 (88) & \\
\hline QSO & & 256 & $180(78)$ & $161(63)$ & \\
\hline NonVar & & & 4791 & $3681(77)$ & \\
\hline Total & & 97912 & $32683(33)$ & $28392(29)$ & \\
\hline
\end{tabular}

Notes. ${ }^{(a)}$ Ratio to the number of variables after crossmatching.

from the EROS-2 database, including these pseudo- or nonperiodic variables in the list is important to exclude these variable types during the selection of periodic variable candidates. There are many BVs in the LMC, some of which show periodic patterns in their light curves. We did not include variable candidates from Marquette et al. (2009), Spano et al. (2011), and Dubath et al. (2012), because they have too few variable candidates of each type. More importantly, using variable sources mainly from a single survey (i.e., OGLE) helps maintain consistency of the training set. The total number of sources in the compiled catalog is 149388 .

We then crossmatched these variables with the entire EROS-2 LMC database with a $3^{\prime \prime}$ search radius that yielded 120825 counterparts, 119480 of which are OGLE periodic variable stars and the remaining 1345 sources are QSOs or BVs. Figure 2 shows a cumulative histogram of distances to the counterparts. To minimize the number of incorrect crossmatches, we only selected sources among these counterparts where the distance between the nearest neighbor and second-nearest neighbor is larger than $3^{\prime \prime}$. After removing these objects, 97912 EROS-2 sources remained.

Even if the known OGLE variables show clear variability in their light curves, it is possible that the crossmatched EROS-2 sources might not show clear variability because of the photometric uncertainty, blending with other sources, different sampling rate, or incorrect crossmatching. To exclude such light curves from a training set, one of us (DWK) visually examined raw- and phase-folded light curves of all 97912 EROS- 2 counterparts. During the visual examination, we removed the light curves without variability or periodicity. Such a pure training set

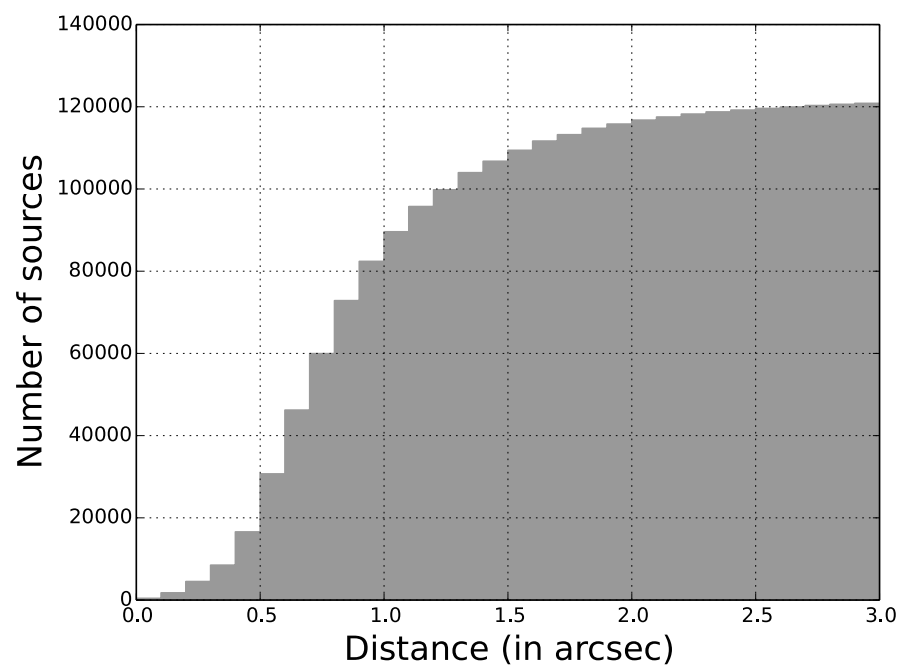

Fig. 2. Cumulative histogram of distances to the EROS-2 counterparts crossmatched with the previously known variables. The largest search radius for crossmatching is $3^{\prime \prime}$. Most of the variables are crossmatched within a $1.5^{\prime \prime}$ search radius.

is important because the goal of this work is to select periodic variable candidates from the entire EROS-2 database, which consists of several tens of millions of light curves. Therefore minimizing false positives mainly consisting of non-variables is the most critical task, which is facilitated by having a highquality training set. Figure 3 shows example light-curves of two RRL variables. We retained light curves with clear variability in 

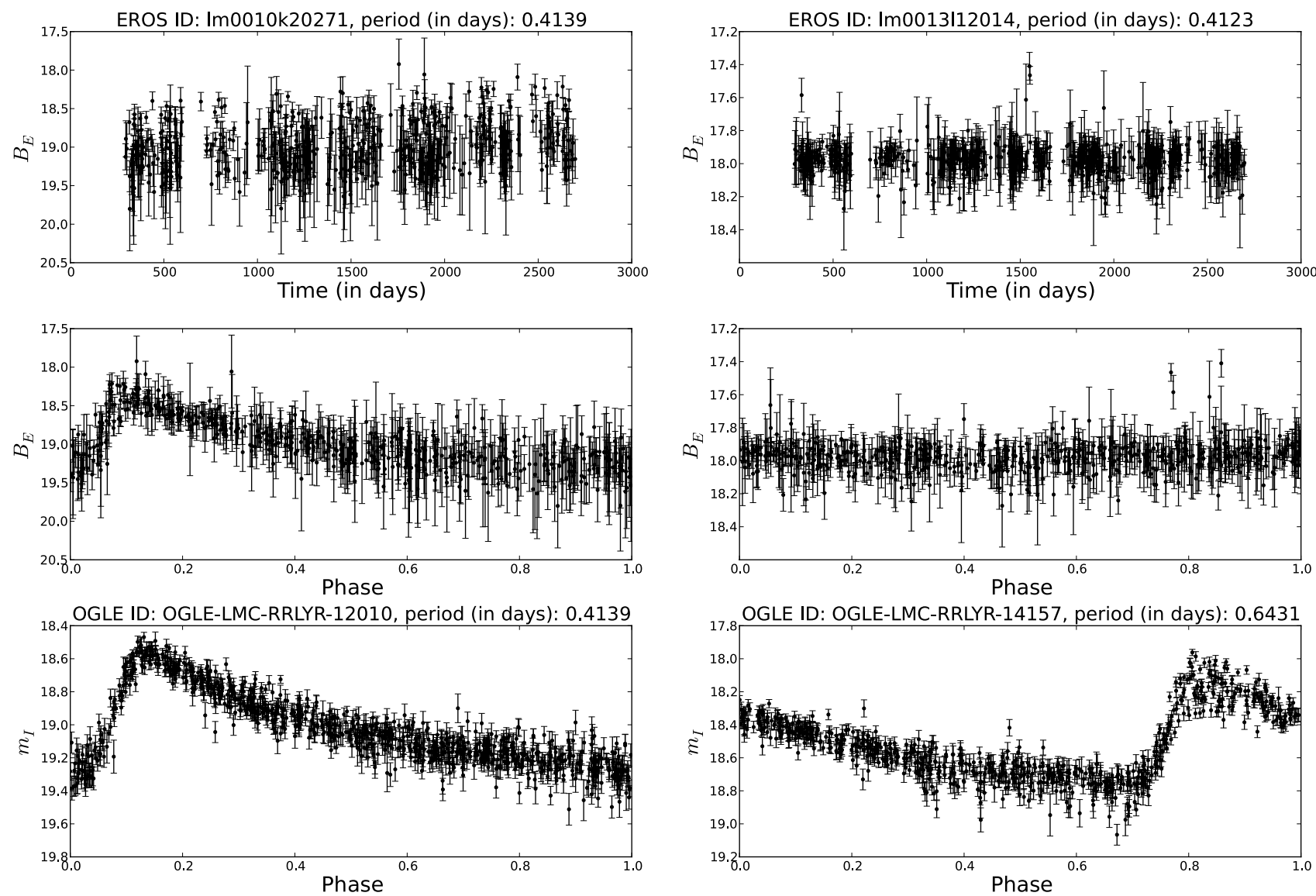

Fig. 3. EROS-2 light curves (top and middle) of two variable stars crossmatched with the OGLE RRLs. Top: raw light curves, middle: phase-folded light curves, left: one RRL variable included in the training set, and right: another RRL variable excluded from the training set. The $x$-axis of raw light curves is in the unit of Heliocentric Julian Date (HJD) - 2450000 . We use this unit as the unit of $x$-axis for a raw light curve throughout this paper. Periods shown in the top panels are derived using the Lomb-Scargle algorithm (Lomb 1976). In the bottom two panels, we show the phase-folded OGLE light curves of the same RRLs. The periods of these light curves are taken from Soszyński et al. (2009b). Although the OGLE RRL in the bottom-right panel shows clear periodic signals, the crossmatched EROS-2 counterpart's light curve (top right and middle right) does not show any variability, which is associated with blending with a nearby star. See text for details.

the EROS-2 light curves, as shown in the left panels. We visually examined an EROS-2 reference image for the source shown in the right panel of Fig. 3 and found that the source is blended with a nearby bright star identified as a RRL variable by the OGLE. This particular case shows that the OGLE differential photometry (the DIA, Alard \& Lupton 1998) is more efficient than the fixed-position photometry of the EROS (the PEIDA, Ansari 1996) to identify variable stars in crowded fields. After this visual removal, we had a set of 27892 periodic variables.

We finally added non-variable sources to this set of visually cleaned light curves by randomly selecting about 5000 light curves that are spread over the whole EROS-2 LMC fields and are not in the 120825 EROS counterparts mentioned in the previous paragraph. Adding non-variables to the training set is critical when selecting variable candidates from the large EROS-2 database because the majority of light curves are non-variables. Therefore a classification model has to be trained in such a way that it excludes non-variables during the variable-star selection. To balance the number of light curves through the EROS-2 magnitude range, we selected about 800 non-variable light curves for each of the magnitude ranges, 13-16, 16-17, 17-18, 18-19, 19-20, and 20-21 $B_{\mathrm{E}}$. If we had randomly selected light curves without the regular magnitude range, there would have been substantially fewer light curves at the brighter end than light curves at the fainter end, which is undesirable because then a trained model might be inefficient in excluding possible non-variables at the bright end. We also visually examined these non-variable light curves and excluded light curves with variability. After adding these non-variables, the final number of light curves in the training set is 32683 .

Without the visual removal of non-variable sources mentioned in the previous paragraph, we observed a significant drop of classification performance, in particular a performance decreased by $\sim 20 \%$ for non-variables (see Sect. 3.4 .2 for the definition of classification performance). This decrease could cause many misclassifications of non-variables into variables, which is not desired because most of the light curves from the EROS-2 database are expected to be non-variables. Nevertheless, even though we did not intentionally remove weak-variability sources, our visual method might result in the rejection of some true variables at the end because of the low signal-to-noise ratio $(\mathrm{S} / \mathrm{N})$ of the variables ${ }^{3}$. In Sect. 3.4.3, we show test results that alleviate these concerns for classifying weak-variability sources.

Table 2 shows the number of variables of each type 1) after the crossmatching; 2) after the visual examination; and 3) after the light-curve refining explained in Sect. 3.2. Although we maintained most of the subclasses of variable types defined from the OGLE catalogs, we merged some subclasses that

\footnotetext{
Signal-to-noise ratio here means the highest amplitude versus noise of a light curve.
} 

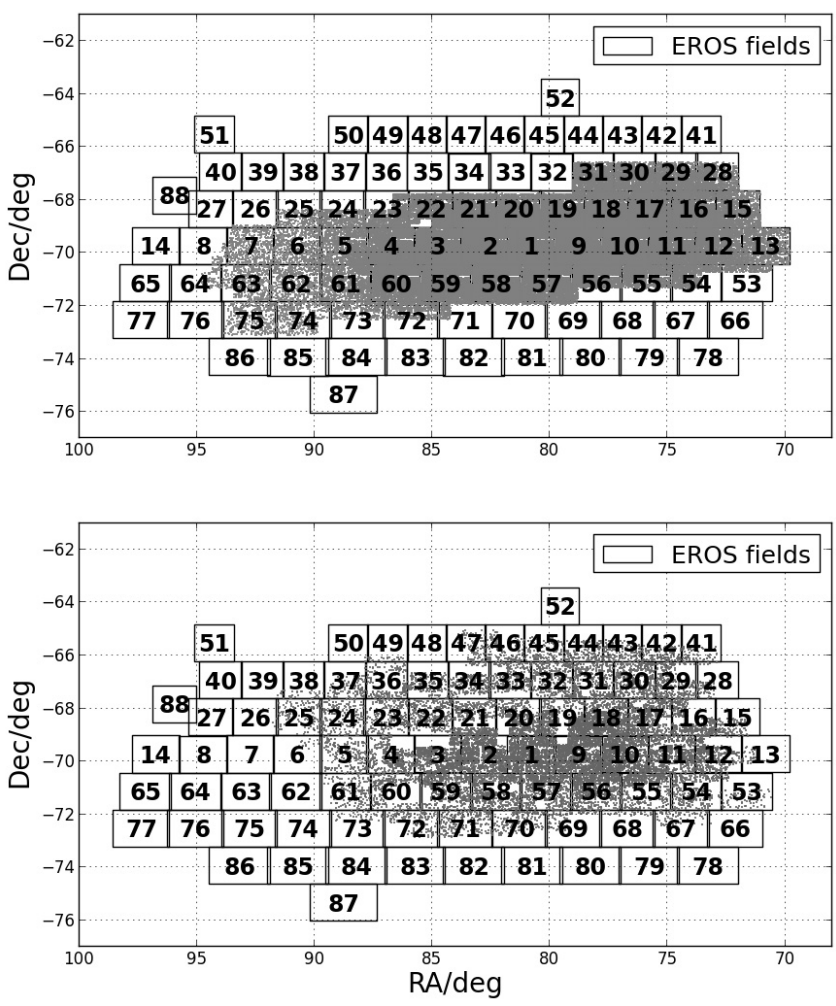

Fig. 4. EROS-2 sources (gray dots) crossmatched with the known OGLE (top) and MACHO (bottom) variable stars in the EROS-2 LMC fields. The rectangles in each panel show the EROS-2 fields. The integer numbers in the rectangles are the EROS-2 field IDs. The $x$ - and $y$-axes are RA and Dec respectively, and both are in J2000.

do not have many sources. For instance, the subclass "Other" of CEPHs includes second-overtone (i.e., 2O), double-mode F/1O (i.e., fundamental-mode and first-overtone), double-mode 1O/2O, double-mode 10/3O (i.e., third-overtone) and triplemode classical CEPHs. One of the EB subclasses, "Other", also includes several types of eclipsing binaries. We also did not separate subclasses of T2CEPHs and DSCTs because of insufficient numbers.

The top panel of Fig. 4 shows the OGLE counterparts before the visual removal superposed on the coverage of the EROS-2 LMC fields. The counterparts are only spread over the central region of the EROS-2 fields. About half of the region is not overlapped by the OGLE counterparts. In addition, we show the crossmatched counterparts with the MACHO variables in the bottom panel of Fig. 4. We used the list of MACHO variables from Kim et al. (2011), which contains about 19k sources including RRLs, CEPHs, EBs, LPVs, and BVs. The MACHO variable study (Alcock 2001) provides one of the richest variable catalogs in terms of the number of variables and variable types along with the OGLE variable studies. As the panel shows, the MACHO variables also do not cover the entire EROS-2 fields. Thus it is clear that there are numerous variable stars that have never been detected by either the OGLE or the MACHO survey.

Note that the training set explained in this section is mainly based on the OGLE variable stars that are spread across the central region of the LMC. Thus it is possible that a classification model built on the training set might be biased to characteristics of the light curves in the central LMC area such as magnitudes, amplitudes, colors, and photometric uncertainties. Nevertheless, training individual classification models adapted to each EROS-2 field's characteristics is beyond the scope of this paper.

\subsection{Refining light curve}

To extract variability features, we used EROS- $2 B_{\mathrm{E}}$-band light curves, which have better photometric accuracy and also more data points than $R_{\mathrm{E}}$-band light curves. This is because the red camera was subject to more technical problems than the blue one during all campaigns. Before extracting variability features from the training set light-curves explained in the previous section, we refined each light curve as follows:

- We removed measurements with magnitudes higher than 22 or with photometric uncertainties higher than 1 mag. This cut removes many measurements of a light curve at the faintend magnitude because such a light curve mainly consists of 99.999 mag values or 9.999 photometric uncertainty values, which indicates that the measurements are unreliable.

- We removed measurements by $3 \sigma$ clipping (no iteration) about the mean, where $\sigma$ is a standard deviation of the light curve. This cut is used to remove fluctuating data points that are occasionally caused by inaccurate photometric measurements. Nevertheless, the cut typically removes less than $5 \%$ of the data points, which does not significantly alter light curves.

We then excluded light curves from the training set if

- there were fewer than 100 measurements;

- the estimated period was spurious, related to the solar day, the moon phase, the sidereal months, and their multiples. We found spurious periods by examining a scatter plot of periods versus period $\mathrm{S} / \mathrm{N}$ (e.g. see the top-left panel in Fig. A.1) and a period histogram.

This reduced the number of light curves reduces from 32683 to 28392 . Most of the excluded light curves were removed by the period criterion. This set of 28392 light curves is the final training set.

\subsection{Variability features}

To identify variability, we used multiple features extracted from the light curves instead of the light curve themselves as the basis for classification. We tested more than 30 features and selected the best 22 features based on their "variable importance", estimated using the random forest algorithm explained in Sect. 3.4.1. We found empirically that using more or fewer features than 22 did not improve classification results. Dubath et al. (2011) also observed that using a large number of features, including inessential or even correlated features did not affect random forest classification results much.

A brief description of each feature is given in Table 3 . Figure 5 shows the estimated variable importance for these 22 features. For details of the definitions of the indices, see Appendix A.

\subsection{Random forest classification model}

\subsubsection{Architecture and training}

To train a classification model using the variability features extracted from the training set light-curves, we used the random forest classification method (Breiman 2001). This has been successfully applied for many astronomical classification and 
Table 3. Variability features.

\begin{tabular}{cl}
\hline \hline Feature & A brief description. For details, see Appendix A. \\
\hline Period & Period derived by the Lomb-Scargle algorithm \\
Period $S / N$ & Signal-to-noise ratio of the derived period \\
$\eta$ & Ratio of the mean of square of successive differences to the variance of data points \\
$\eta_{B-R}$ & $\eta$ over a $B_{\mathrm{E}}-R_{\mathrm{E}}$ light curve \\
$\psi^{\eta}$ & $\eta$ over a phase-folded light curve \\
$\eta^{e}$ & Modified $\eta$ \\
$B_{\mathrm{E}}$ & Mean magnitude of $B_{\mathrm{E}}$ band \\
$B_{\mathrm{E}}-R_{\mathrm{E}}$ & Color. Difference of mean magnitude of $B_{\mathrm{E}}$ and $R_{\mathrm{E}}$ bands \\
$Q_{3-1}$ & 3rd quartile (75\%) -1 st quartile $(25 \%)$ \\
$Q_{3-1 \mid B-R}$ & 3rd quartile (75\%) -1 st quartile (25\%) over a $B_{\mathrm{E}}-R_{\mathrm{E}}$ light curve \\
Cusum & Range of cumulative sum \\
$\psi^{\mathrm{CS}}$ & Cusum over a phase-folded light curve \\
$\sigma$ & Standard deviation \\
$\gamma_{1}$ & Skewness \\
$\gamma_{2}$ & Kurtosis \\
$J$ & Stetson $J$ index \\
$K$ & Stetson $K$ index \\
$K_{\mathrm{AC}}$ & Stetson $K$ index derived over an autocorrelation function of a light curve \\
$R_{21}$ & 2nd-to-1st amplitude ratio derived using the Fourier decomposition \\
$R_{31}$ & 3rd-to-1st amplitude ratio derived using the Fourier decomposition \\
$\phi_{21}$ & Difference between 2nd and 1st phases derived using the Fourier decomposition \\
$\phi_{31}$ & Difference between 3rd and 1st phases derived using the Fourier decomposition \\
\hline
\end{tabular}

Notes. We used EROS $B_{\mathrm{E}}$-band light curves (either raw- or phase-folded) to derive these features, except for $J, Q_{3-1 \mid B-R}$ and $\eta_{B-R}$, which needs both $B_{\mathrm{E}^{-}}$and $R_{\mathrm{E}}$-band light curves. See Appendix A for details.

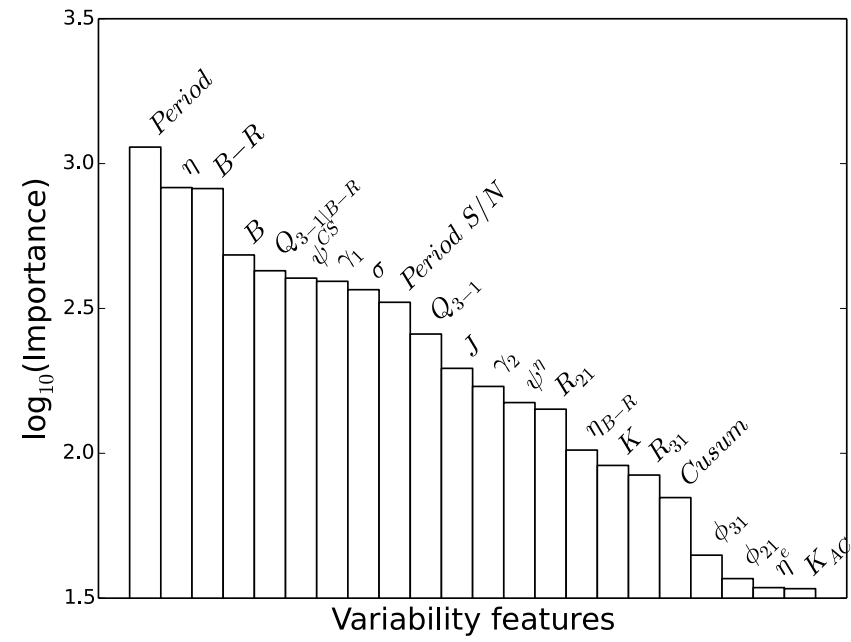

Fig. 5. Variable importance of the variability features as determined by the random forest algorithm considering the variable subclasses. Higher importance features are more relevant for classification than lower importance features. A brief summary of these features is given in Table 3.

regression problems (e.g. Carliles et al. 2010; Dubath et al. 2011; Richards et al. 2011; Pichara et al. 2012). Random forest uses multiple decision trees (Quinlan 1993) and the bagging (Breiman 1996) to train a classification model. Each decision tree is trained using a subset of features that are randomly selected from all features (here the 22 variability features mentioned in the previous section). Random forest then chooses a class for a sample that has the most votes of the all decision trees. In principle, random forest does not need to do crossvalidation to estimate the classification error because each tree is constructed based on the bootstrap sample from the training set, where two thirds of the samples are used to train the tree and the remaining one third of samples, which are out-of-bag (oob) samples, is used to estimate an error, which is an oob error. The process of constructing each decision tree is as follows:

1. Generate $N$ light curves randomly selected from the training set with replacement, where $N$ is the total number of light curves in the training set, which is called bootstrap aggregating (bagging). This sample is used to train the tree.

2. At each node, randomly select $m$ features from the all features, where $m<M . M$ is the total number of features. The $m$ value is fixed during the training.

3. These randomly selected $m$ features are used to split the node. At each node, every possible split is tested, and then a feature for the best split is chosen. The tree is grown to its full extent without pruning. Each leaf node of the tree returns a single sample.

4. Repeat $t$ times the above processes, where $t$ is the number of decision trees.

While training a random forest model, one needs to set two free parameters, $t$ and $m$, which is the number of decision trees and the number of features, respectively. We briefly explain the parameter selection in the following section.

We can use random forest to estimate the variable importance relevant to classification as follows:

1. train a normal random forest model, $R F_{\text {normal }}$;

2. train another random forest model after randomly permuting values of a feature among the training set, $R F_{\text {permute }}$;

3. during the training processes, calculate differences in oob error between the trees from the $R F_{\text {normal }}$ and the $R F_{\text {permute }}$. 
D.-W. Kim et al.: The EPOCH Project. I.

Table 4. Confusion matrix of the random forest model without considering subclasses.

\begin{tabular}{|c|c|c|c|c|c|c|c|c|c|c|}
\hline Class & DSCT & RRL & CEPH & T2CEPH & EB & LPV & BV & QSO & Nonvar & $\begin{array}{c}\text { Recall } \\
(\%)\end{array}$ \\
\hline$\overline{\mathrm{DSCT}}$ & 253 & 1 & & & & & & & 1 & 99.2 \\
\hline RRL & 3 & 2088 & 4 & 2 & 16 & & & & & 98.8 \\
\hline CEPH & & 10 & 1094 & 3 & 12 & & 1 & & 1 & 97.6 \\
\hline T2CEPH & & & 8 & 37 & 10 & 1 & & & & 66.1 \\
\hline EB & 1 & 11 & 6 & & 1652 & 20 & & & 14 & 96.9 \\
\hline LPV & & & & 1 & 6 & 6642 & 1 & 1 & 12 & 99.7 \\
\hline BV & & & & & 2 & & 353 & 1 & 6 & 97.5 \\
\hline QSO & & & & & & 1 & 2 & 71 & 7 & 87.7 \\
\hline NonVar & 2 & 1 & & & 6 & 12 & 5 & 4 & 1812 & 98.4 \\
\hline $\begin{array}{c}\text { Precision } \\
(\%)\end{array}$ & 97.7 & 99.0 & 98.4 & 86.0 & 96.9 & 99.5 & 97.5 & 92.2 & 97.8 & $98.6,98.6^{a}$ \\
\hline
\end{tabular}

Notes. Each row is a true class and each column is a predicted class. Bold numbers show the true positives. ${ }^{(a)}$ Average weighted recall and precision by the proportion of classes in the training samples.

Average the differences over all trees and normalize the average differences by its standard deviation derived from the differences. This averaged and normalized difference is a measure of the importance of the feature.

Thus stronger features generally have higher variable importance values. The derived variable importance of the 22 variability features is given in Fig. 5.

Random forest results can be used to estimate class probabilities for each light curve based on a simple voting strategy,

$P_{\text {class }}(i)=\frac{n_{\text {class }}(i)}{N}$,

where $P_{\text {class }}$ is the probability of a class for a sample, $n_{\text {class }}$ is the number of trees that identify the sample as class, and $N$ is the total number of trees. For each light curve, we accepted the class corresponding to the highest probability among probabilities of all classes. We did not modify the probability according to class population in the training set since it is not the true population.

\subsubsection{Training a classification model and performance optimization}

Although random forest can internally estimate the classification error, as mentioned in the previous section (oob error), we separated the training set mentioned in Sect. 3.1 into two sets of samples to 1) measure performance; and 2) find the best $t$ (the number of trees) and $m$ (the number of features). The samples consist of $50 \%$ of the training set ( 14000 light curves) to train the model and the remaining $50 \%$ to estimate the trained model's classification performance. We call these two set $S_{1}$ and $S_{2}$. We assessed the classification performance using a weighted average of recall and precision by class size in the training set. The recall and precision are defined as

recall $=\frac{N_{\mathrm{TP}}}{N_{\mathrm{TP}}+N_{\mathrm{FN}}}$, precision $=\frac{N_{\mathrm{TP}}}{N_{\mathrm{TP}}+N_{\mathrm{FP}}}$,

where $N_{\mathrm{TP}}$ is the number of the true positives, $N_{\mathrm{FP}}$ is the number of the false positives, and $N_{\mathrm{FN}}$ is the number of the false negatives. To select the best $t$ and $m$, we trained a random forest model for all possible combinations of $t$ and $m$ with $20 \leq t \leq 400$ and $5 \leq m \leq 21$ with a step size of 20 and 2, respectively. We then applied each trained model to $S_{2}$ to measure the model's performance. Finally, we chose the $t$ and $m$ with the best performance. Figure 6 shows the classification performance for each

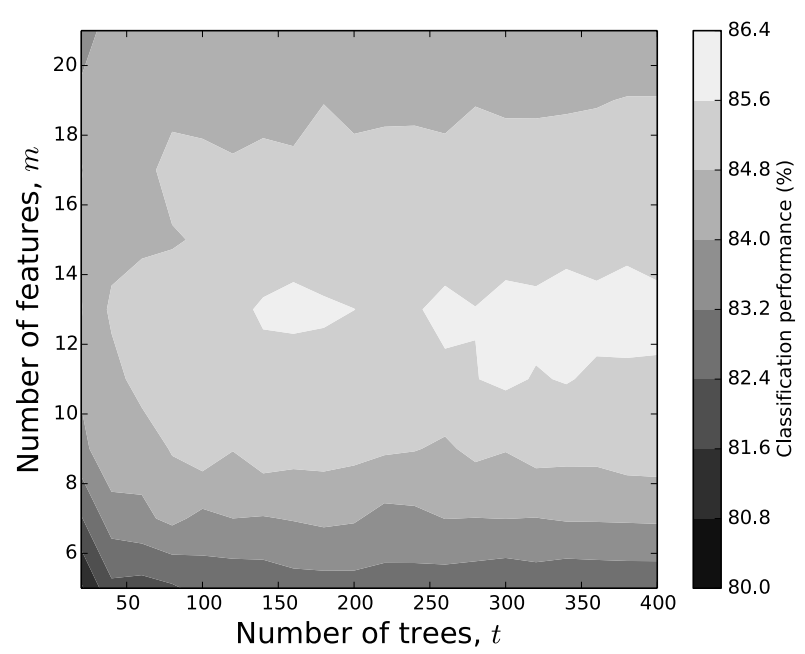

Fig. 6. Classification performance according to the number of trees, $t$, and the number of features, $m$. Performance degrades when $m>14$, which might be caused by overfitting of a classification model.

combination of $t$ and $m$. The classification performance through this parameter space is relatively stable, as shown in the figure.

Tables 4 and 5 show the performance of the trained models using the optimized parameters, $t$ and $m$. In Table 4, we present the confusion matrix of the model trained with only superclasses (i.e., without considering subclasses of variable types). We see that recall and precision for each superclass is higher than $95 \%$, and average recall and precision is about $99 \%$. Only the T2CEPH class shows low recall and precision. This is because 1) the number of variables in this class is substantially lower than that of the other classes; and 2) the variability characteristics of T2CEPHs are similar to other classes (i.e., sinusoidal pattern of the light curves). Table 5 shows the recall and precision of the model trained with the subclasses ${ }^{4}$. The average recall and precision drop to $87 \%$, not because of the misclassification between superclasses, but mainly because of the misclassification of subclasses within a superclass. This is not surprising since the classification of each subclass within a same superclass is harder than the separation of superclasses. It is also possible that the OGLE classification is not sufficiently accurate, especially

4 Due to the length of the confusion matrix, we do not show it but just show the recall and precision for each class. The confusion matrix is available in Appendix B. 
Table 5. Recall and precision of the random forest model considering subclasses.

\begin{tabular}{|c|c|c|c|}
\hline Class & Subclass & $\begin{array}{c}\text { Recall } \\
(\%)\end{array}$ & $\begin{array}{c}\text { Precision } \\
(\%)\end{array}$ \\
\hline DSCT & & 97.6 & 98.0 \\
\hline \multicolumn{4}{|l|}{$\mathrm{CEPH}$} \\
\hline & $\mathrm{F}$ & 97.3 & 97.7 \\
\hline & 10 & 91.1 & 91.6 \\
\hline & Other & 71.3 & 73.1 \\
\hline \multicolumn{4}{|l|}{ RRL } \\
\hline & $a b$ & 99.6 & 99.0 \\
\hline & $\mathrm{c}$ & 95.9 & 87.4 \\
\hline & $\mathrm{d}$ & 65.1 & 93.3 \\
\hline & $\mathrm{e}$ & 83.1 & 85.5 \\
\hline \multicolumn{4}{|l|}{ EB } \\
\hline & $\mathrm{EC}$ & 52.6 & 70.1 \\
\hline & ED & 88.9 & 79.9 \\
\hline & ESD & 73.0 & 65.0 \\
\hline & $\mathrm{ED}+\mathrm{ESD}$ & 5.6 & 50.0 \\
\hline & Other & 11.0 & 44.4 \\
\hline \multicolumn{4}{|l|}{ LPV } \\
\hline & Mira AGB O & 82.1 & 86.9 \\
\hline & OSARG AGB C & 57.0 & 71.9 \\
\hline & OSARG AGB O & 80.8 & 76.1 \\
\hline & OSARG RGB C & 13.0 & 30.0 \\
\hline & OSARG RGB O & 84.4 & 83.4 \\
\hline & SRV AGB C & 88.2 & 85.3 \\
\hline & SRV AGB O & 84.4 & 84.9 \\
\hline T2CEPH & & 71.4 & 88.9 \\
\hline BV & & 96.1 & 97.2 \\
\hline QSO & & 92.5 & 91.4 \\
\hline NonVar & & 98.2 & 97.6 \\
\hline Average recall and precision ${ }^{a}$ & & 86.7 & 86.3 \\
\hline
\end{tabular}

Notes. ${ }^{(a)}$ Average weighted by the proportion of classes in the training samples.

for EBs and LPVs, as the OGLE papers Soszyński et al. (2009a) and Graczyk et al. (2011) indicate.

In Fig. 7 we show two example EB light curves. The probability of being in the EB class for the light curve in the left panel is 1.0, while the probability for the light curve in the right panel is 0.38 . As the figure clearly shows, the light curve with a high probability has a much stronger signal in the raw- and phasedfolded light curve than the one on the right. The estimated periods for EBs are probably half of the true periods, particularly when there is little difference between amplitudes of primary and secondary eclipses. However, it would not significantly affect the classification performance because the model is trained not only on period, but other variability features as well. In Fig. 8, we show the distribution of probability of related class for $S_{2}$ (probability, for short). About half of the light curves' probabilities are higher than 0.9. Conversely, there are fewer samples with low probabilities. This is because we visually removed light curves with no or feeble variability when constructing the training set.

The final model is trained using the 22 variability features, the entire training set consists of 28392 light curves (i.e., $S_{1}+$ $S_{2}$ ) and all the variable subclasses, where $t=160$ and $m=$ 13. This model was used to select and classify periodic variable candidates from the EROS-2 database.

\subsubsection{Evaluation of the trained model}

As mentioned in Sect. 3.1, we removed 65229 (i.e., 97912-32683; see Table 2) sources from the training set by visual examination. Thus it is possible that we accidentally excluded true variables with low-level variability. In this section, we assess the classification quality of the trained model for weak variability using these visually removed sources.

We first refined these 65229 light curves as explained in Sect. 3.2, which yielded 38201 sources. Most of the light curves were removed by the period criterion. Figure 9 shows histograms of variability features of 1) non-variables in the training set; 2) RRLs in the visually removed 38201 sources; and 3) RRLs in the training set. The set of RRLs that were removed is almost entirely overlapped by the RRL training set, implying that this visual removal has not significantly biased the training set. Figure 10 shows histograms of the variability features of LPVs. LPVs that were visually removed lie between the non-variables and the final LPV training set. Objects in the left part of the histogram for the removed LPVs have parameters similar to those of the non-variables in the training set, which means that they are probably also non-variables. The right half is similar to the LPVs in the training set, which indicates that they are probably weakly variable LPVs mistakenly excluded by the visual inspection (an inevitable consequence of trying to build a very clean training set). Nevertheless, as the figure shows, the training-set LPVs cover the right half of the histogram well. We have confirmed that other types of variables show a similar distribution to either Figs. 9 or 10.

We then applied the trained model to these 38201 sources. Among these sources, 12875 sources are classified as nonvariables and the remaining 25326 sources are classified as variables. Figure 11 shows the probability distribution of the variable 

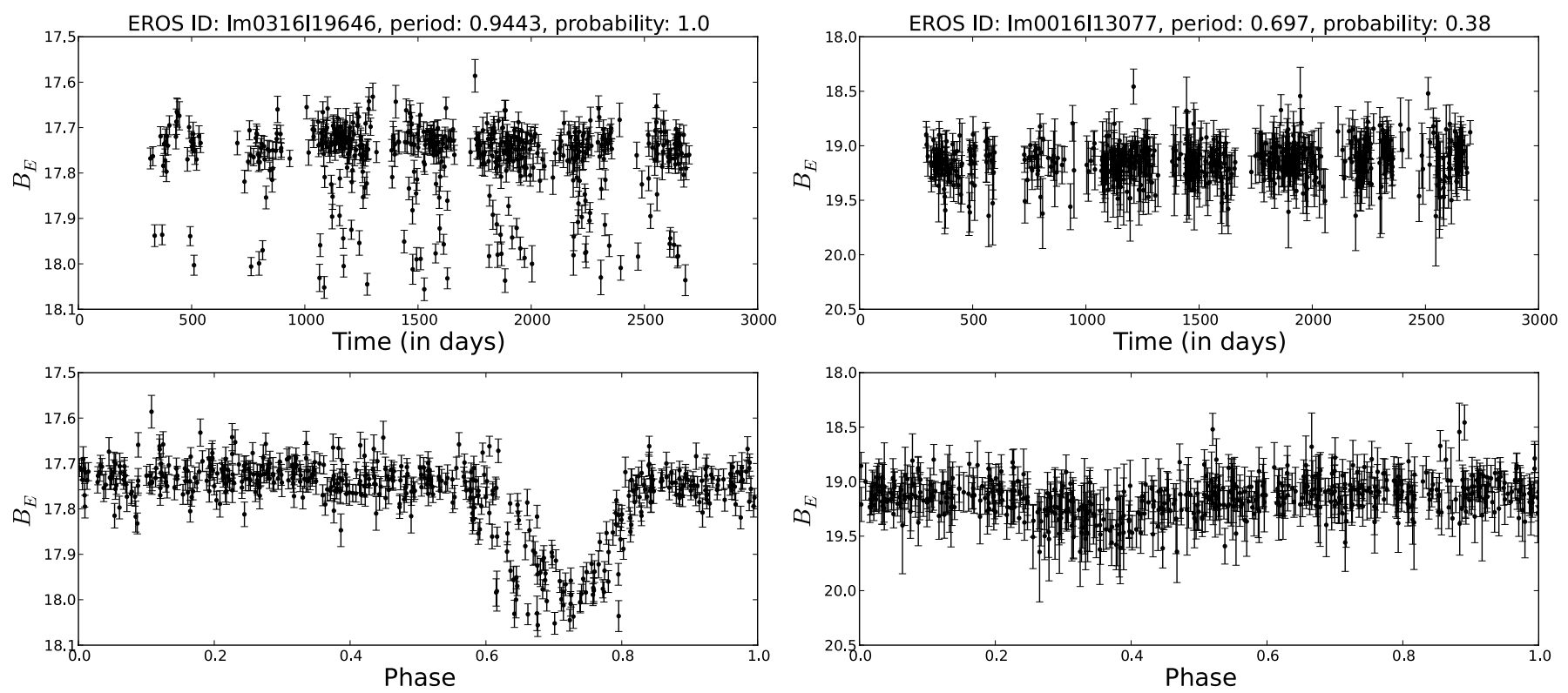

Fig. 7. Example light curves of two EB variables with a high (left panel) and low probability (right panel). The two left (right) panels are light curves of a same star. The top panels are raw light curves and the bottom panels are phase-folded light curves. The light curve with a high probability shows much stronger and clearer transit signals than the other light curve.

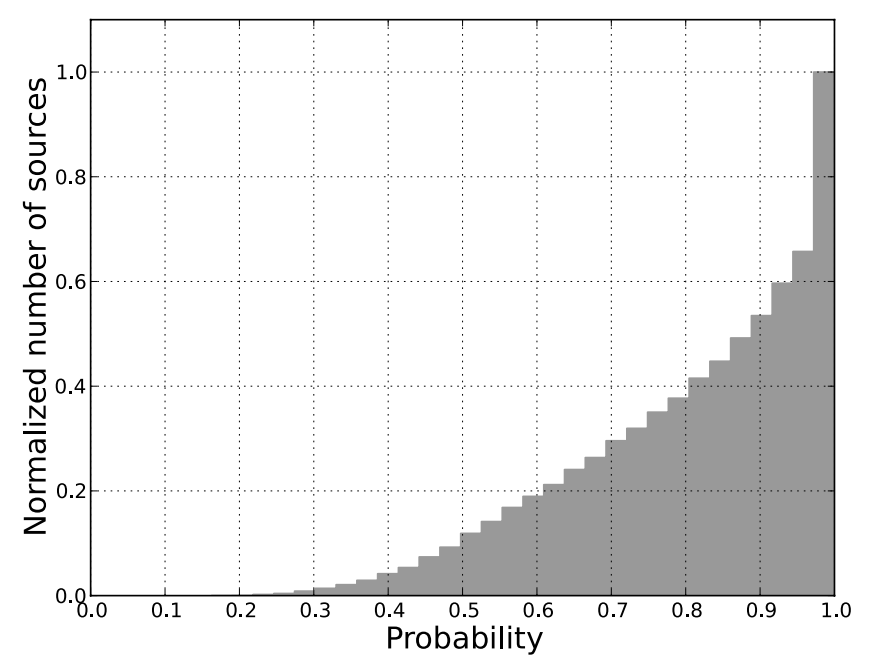

Fig. 8. Cumulative histogram of probabilities of related class for $S_{2}$. There are more high-probability than low-probability light curves. About half of the light curves have probabilities higher than 0.9.

Table 6. Superclass recall and precision for the removed sources.

\begin{tabular}{crc}
\hline \hline Class & $\begin{array}{r}\text { Recall } \\
(\%)\end{array}$ & $\begin{array}{c}\text { Precision } \\
(\%)\end{array}$ \\
\hline DSCT & 100.0 & 80.3 \\
CEPH & 20.7 & 37.5 \\
RRL & 94.7 & 98.6 \\
EB & 96.4 & 93.0 \\
LPV & 99.7 & 99.9 \\
\hline
\end{tabular}

sources. A relatively larger portion of sources has low probabilities than in Fig. 8. This is not surprising since these sources probably have weak variability and thus low probabilities. Table 6 shows recall and precision for superclass classification. The recall of $\mathrm{CEPH}$ is much worse than others. Most of the CEPHs
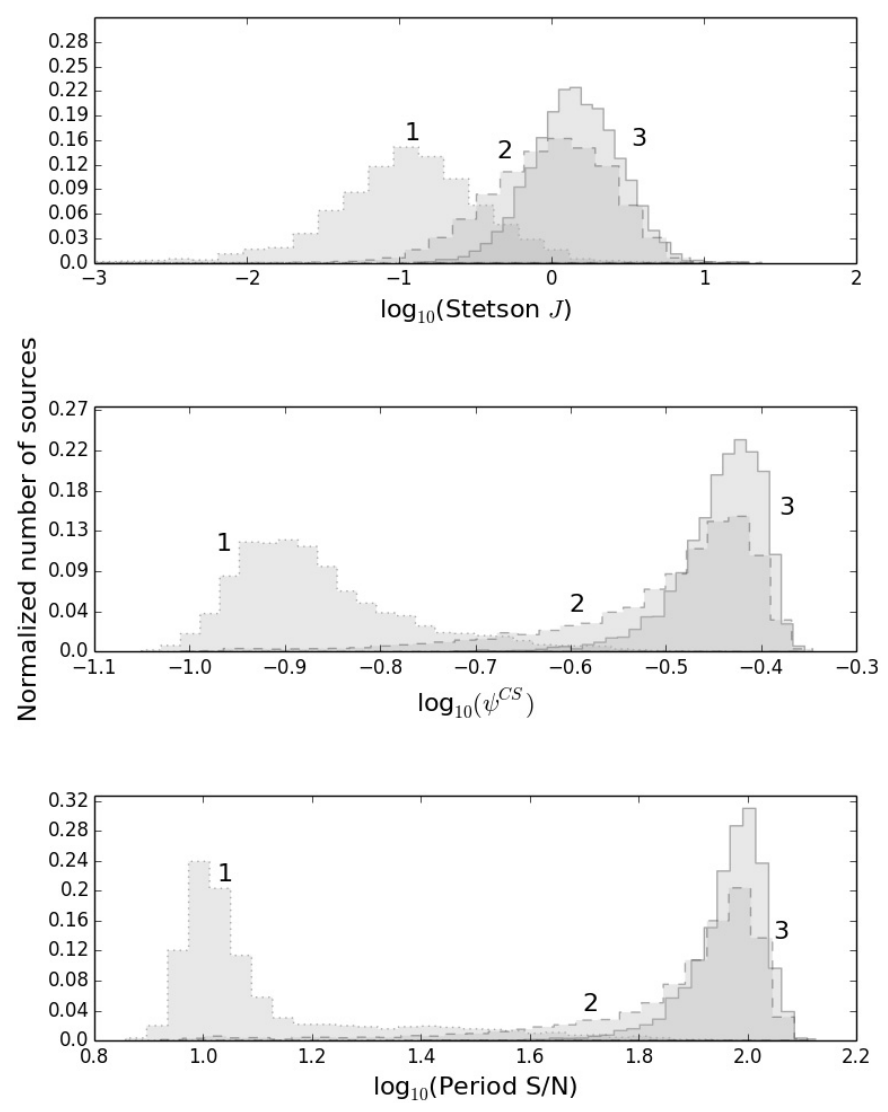

Fig. 9. Histograms of three variability features, Stetson $J, \psi^{\mathrm{CS}}$, and period $\mathrm{S} / \mathrm{N}$ (from top to bottom). Each panel shows three histograms, each of which is a histogram of 1) non-variables (dotted line) in the training set; 2) the visually removed RRLs (dashed line); and 3) RRLs (solid line) in the training set. See text for details.

are classified as RRL c type stars that generally show a similar variability pattern with CEPHs - slow increase and decrease 

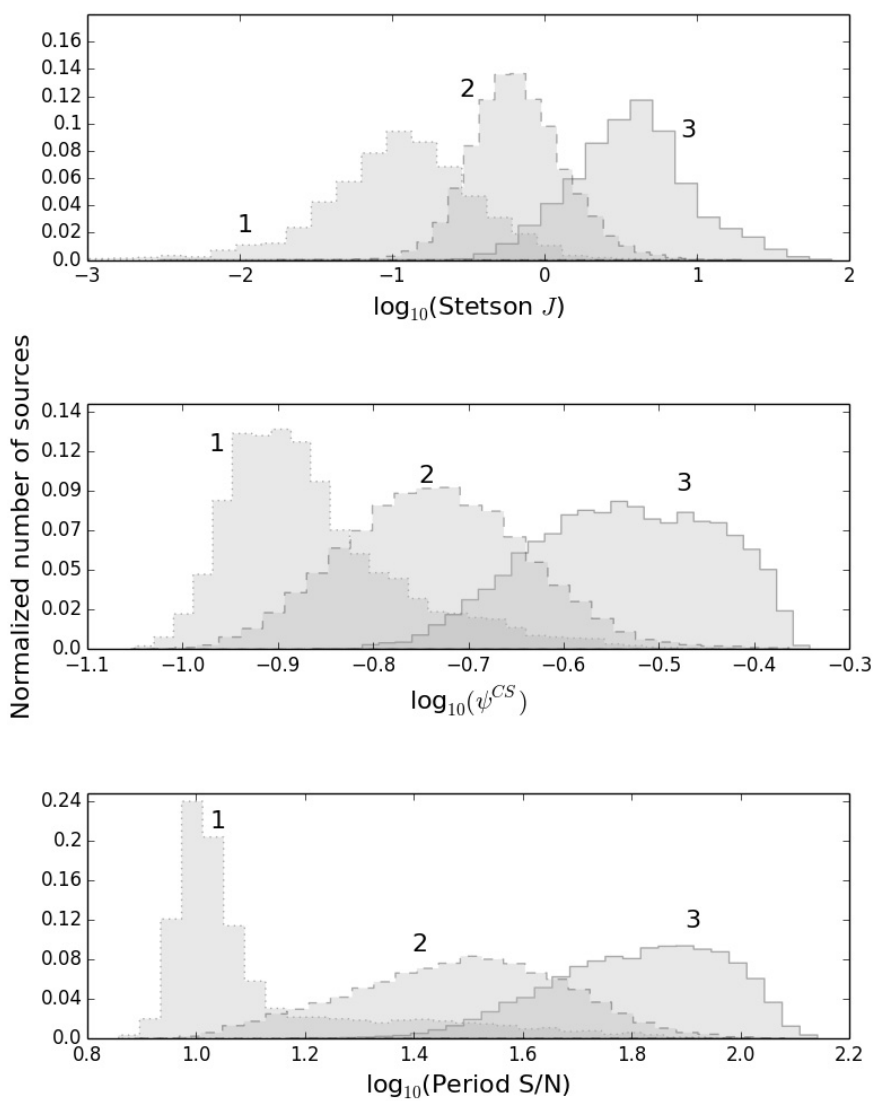

Fig. 10. Histograms of three variability features, Stetson $J, \psi^{\mathrm{CS}}$, and period S/N (from top to bottom). Each panel shows three histograms, each of which is a histogram of 1) non-variables (dotted line) in the training set; 2) the visually removed LPVs (dashed line); and 3) LPVs (solid line) in the training set. See text for details.

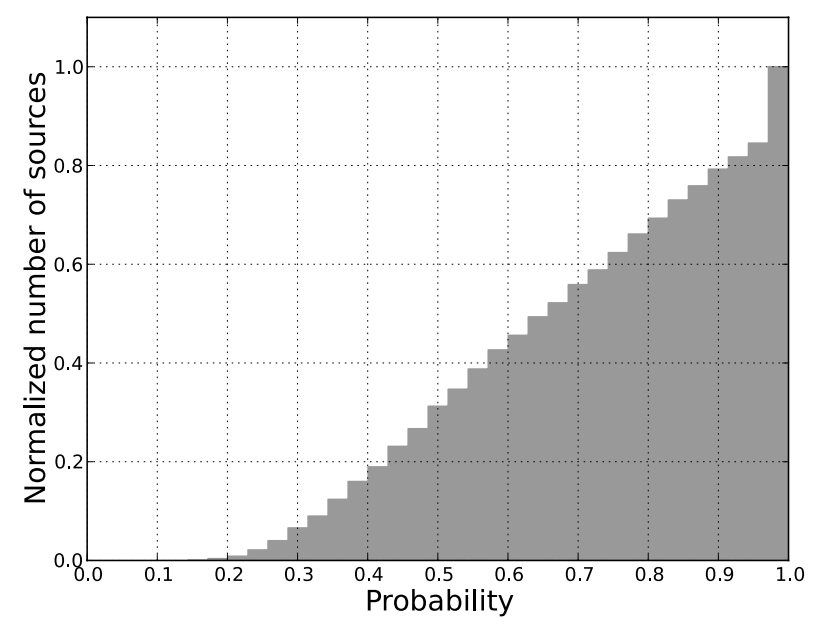

Fig. 11. Cumulative histogram of probabilities of the visually removed sources that are identified as variable stars by the trained model. Compared with Fig. 8, there are more low-probability light curves.

of flux during its phase (i.e., sinusoidal). We visually examined these misclassified CEPHs and found that their light curves are not clearly distinguishable from RRLs. Nevertheless, the high recall and precision of other periodic variable classes indicates that the trained model is successful in classifying weak variability sources. We also show recall and precision for the subclass classification of these variables in Table 7. The recall and precision of EBs and LPVs are poorer than others, which might
Table 7. Subclass recall and precision for the removed sources.

\begin{tabular}{ccrr}
\hline \hline Superclass & Subclass & $\begin{array}{r}\text { Recall } \\
(\%)\end{array}$ & $\begin{array}{r}\text { Precision } \\
(\%)\end{array}$ \\
\hline CEPH & 10 & 12.5 & 42.9 \\
& Other & 0.0 & 0.0 \\
RRL & & & \\
& ab & 93.3 & 99.4 \\
& $\mathrm{c}$ & 90.1 & 87.3 \\
& $\mathrm{~d}$ & 70.6 & 86.0 \\
EB & $\mathrm{e}$ & 80.2 & 74.1 \\
& & & \\
& EC & 40.3 & 26.5 \\
& ED & 85.6 & 75.4 \\
& ESD & 43.0 & 56.1 \\
& ED+ESD & 7.3 & 10.2 \\
LPV & Other & 0.0 & 0.0 \\
& & & \\
& OSARG AGB C & 82.7 & 11.5 \\
& OSARG AGB O & 76.4 & 50.5 \\
& OSARG RGB C & 15.8 & 0.4 \\
& OSARG RGB O & 37.7 & 95.2 \\
& SRV AGB C & 38.3 & 38.6 \\
& SRV AGB O & 75.3 & 16.2 \\
\hline
\end{tabular}

Notes. We do not show some subclasses (e.g. CEPH F) whose recall or precision we were unable to calculate because of the lack of the subclasses either in the OGLE classification or in the trained model's classification.

be associated with either 1) misclassification due to weak variability; or 2) incomplete OGLE subclass classification (e.g. see Soszyński et al. 2009a; Graczyk et al. 2011).

We visually examined the light curves with probabilities higher than 0.9 (see Fig. 11) and found that the majority of them show strong variability, but were mistakenly excluded during the visual removal. We trained another classification model after including these high-probability sources into the training set to check whether or not these samples help improve the model performance. We found that the newly trained model showed almost identical performance to the original model.

In this section, we assessed the classification quality of the trained model for weak variability. Although some true variables might be mistakenly removed during the visual inspection because of the low level of variability, most of the removed sources are relatively well covered by the training set, as shown in Figs. 9 and 10. Moreover, Tables 6 and 7 show that the model has a fairly acceptable classification quality for such sources. Nevertheless, note that the goal of this work is to select variables from the 29 million EROS-2 sources where the majority of the sources are expected to be non-variables. Therefore minimizing possible false positives is the most critical task. As mentioned in Sect. 3.1, we observed a substantial decrease of classification performance without this visual removal.

\section{Variable candidates from the EROS-2 LMC database}

We refined all light curves as depicted in Sect. 3.2 from the entire EROS-2 LMC database ${ }^{5}$, extracted 22 variability features from them, and applied the trained model. This yielded 150115 variable candidates. From these light curves, we removed light

5 About $50 \%$ of the EROS-2 light curves were removed by the criteria. Five sixths of them were removed by the period criterion. 
Table 8. Number of variable candidates selected from the EROS-2 LMC fields.

\begin{tabular}{|c|c|c|c|}
\hline Superclass & Subclass & $\mathrm{C} 1^{a}$ & $\bar{C} 2^{b}$ \\
\hline DSCT & & 2481 & 1906 \\
\hline \multicolumn{4}{|l|}{$\mathrm{CEPH}$} \\
\hline & $\mathrm{F}$ & 1993 & 246 \\
\hline & 10 & 1527 & 318 \\
\hline & Other & 343 & 74 \\
\hline \multicolumn{4}{|l|}{ RRL } \\
\hline & $a b$ & 16407 & 3599 \\
\hline & $\mathrm{c}$ & 5367 & 1904 \\
\hline & d & 968 & 311 \\
\hline & $\mathrm{e}$ & 1636 & 793 \\
\hline \multicolumn{4}{|l|}{ EB } \\
\hline & $\mathrm{EC}$ & 6120 & 5159 \\
\hline & $\mathrm{ED}$ & 13372 & 7216 \\
\hline & ESD & 13970 & 10627 \\
\hline & $\mathrm{ED}+\mathrm{ESD}$ & 674 & 422 \\
\hline & Other & 11471 & 11138 \\
\hline \multicolumn{2}{|l|}{ LPV } & 918 & 313 \\
\hline & Mira AGB O & 454 & 99 \\
\hline & OSARG AGB C & 3538 & 883 \\
\hline & OSARG AGB O & 15192 & 3992 \\
\hline & OSARG RGB C & 1015 & 217 \\
\hline & OSARG RGB O & 8863 & 3031 \\
\hline & SRV AGB C & 3884 & 1122 \\
\hline & SRV AGB O & 6712 & 1737 \\
\hline T2CEPH & & 329 & 178 \\
\hline Total & & 117234 & 55285 \\
\hline
\end{tabular}

Notes. ${ }^{(a)}$ After applying $B_{\mathrm{E}} \leq 20$ and period $S / N \leq 20$ cuts. ${ }^{(b)}$ After additional removal of the known OGLE and MACHO variables.

curves with $B_{\mathrm{E}}$ fainter than 20 , which is around the limiting magnitude. 27224 light curves were removed by the magnitude cut. We also visually examined some of the variable-candidate light curves and found that some light curves with a period $\mathrm{S} / \mathrm{N}$ lower than 20 are likely false positives (i.e., non-variables). For instance, the top-left panel of Fig. A.1 shows a scatter plot of period and period $\mathrm{S} / \mathrm{N}$ of the training-set light curves (symbols). The panel also shows a contour line of $\sim 550 \mathrm{k}$ field sources from the EROS-2 LMC database. The majority of the non-variable sources (gray circles) in the training set and the field sources have a period $\mathrm{S} / \mathrm{N}$ lower than 20. Figures 9 and 10 show the same distribution. Low-period $\mathrm{S} / \mathrm{N}$ can be caused by large photometric uncertainties, relatively weak periodic signals, insufficient number of measurements, etc. Although not every source with $B_{\mathrm{E}}>20$ or period $S / N<20$ is a non-variable, we removed all such sources to minimize the number of potential false positives $^{6} .5657$ light curves were removed by the period $\mathrm{S} / \mathrm{N}$ cut. After this removal, the number of variable candidates is 117234 , which is about $0.44 \%$ of the total sources in the EROS-2 LMC database. In Table 8, we show the number of variable candidates after these cuts.

Among these 117234 variable candidates, we found that 58069 variable candidates are previously known OGLE variables. We also crossmatched the 117234 EROS-2 variable candidates with the known MACHO variables. The list of known MACHO variables is taken from Kim et al. (2011) and contains 19097 variables. Among these 19097 MACHO variables, 16543 are crossmatched with the EROS-2 variable candidates.

\footnotetext{
6 The training set light curves of variable sources show strong variability regardless of their $B_{\mathrm{E}}$ magnitudes or period $\mathrm{S} / \mathrm{N}$.
}

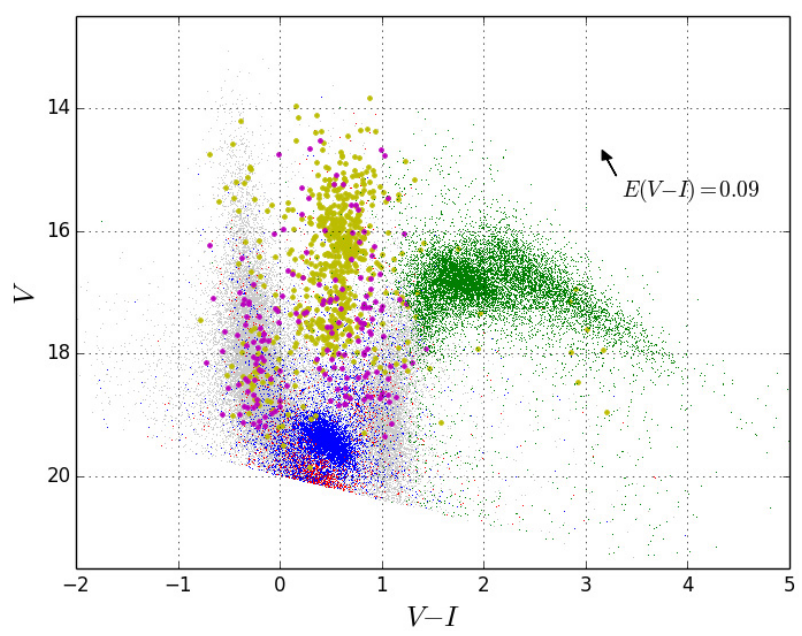

Fig. 12. Color-magnitude diagram of the newly detected 55285 variable candidates. DSCTs: red dots, RRLs: blue dots, EBs: gray dots, LPVs: green dots, CEPHs: yellow circles and T2CEPHs: magenta circles. The magnitudes are not corrected for interstellar extinction. The arrow represents average interstellar extinction of $E(V-I)=0.09$ for the LMC (Haschke et al. 2011). The typical distance modulus to the LMC is 18.5 mag (Tisserand et al. 2009).

We excluded EROS-2 DSCT and T2CEPH candidates during the crossmatching because the list of the known MACHO variables does not have these classes. Table 8 shows the number of variable candidates of each type after removing the known OGLE and MACHO variables. Figure 12 is a color-magnitude diagram of the new EROS-2 variable candidates. We transformed EROS- 2 bands $B_{\mathrm{E}}$ and $R_{\mathrm{E}}$ to the standard $V$ Johnson and I Cousins broadband (Tisserand et al. 2009). Figure 13 shows examples of phase-folded light curves of six new variable candidates. There is clear and strong variability in the phase-folded light curves. In Fig. 14, we show period histograms of each variable type. It is well-known that different types of periodic variable stars have different ranges of periods such as DSCTs: 0.02-0.25 days (Poleski et al. 2010), RRLs: 0.2-0.9 days (Alcock et al. 1996) and CEPHs: days to months (Soszyński et al. 2008a). We visually examined the two T2CEPHs with periods longer than 1000 days and found that they might be misclassified LPVs. The probabilities of these two T2CEPHs are relatively low, $18 \%$ and $25 \%$. The histogram of the EB periods in Fig. 14 shows two populations, one with periods shorter than 20 days, another with periods longer than 20 days. We confirmed that the shorter period EBs are on the main sequence, while the longer period EBs are on the red giant branch, which is a known behavior (Graczyk et al. 2011). Figure 15 shows the probabilities of the candidates. Most of DSCTs, RRLs, and CEPHs have probabilities higher than $90 \%$. In contrast, many T2CEPHs, EBs, and LPVs have probabilities lower than $50 \%$. In particular, about two thirds of EBs have probabilities lower than 50\%, which is probably caused by either misclassification within EB subclasses or misclassification between superclasses. Nevertheless, we visually examined these low-probability candidates and confirmed that almost all of them show variability in their light curves.

Figures 16 to 18 show the relationships between features of each subclass of RRLs, CEPHs, and LPVs in the newly detected 55285 variable candidates. We do not show EBs since they are mixed in most of the 2D-plane of variability features. This is not surprising since the subclass classification performance of EBs is relatively poor (see Table 5). Figure 16 shows period and amplitude ratio, $R_{21}$, of the RRL variable candidates; 

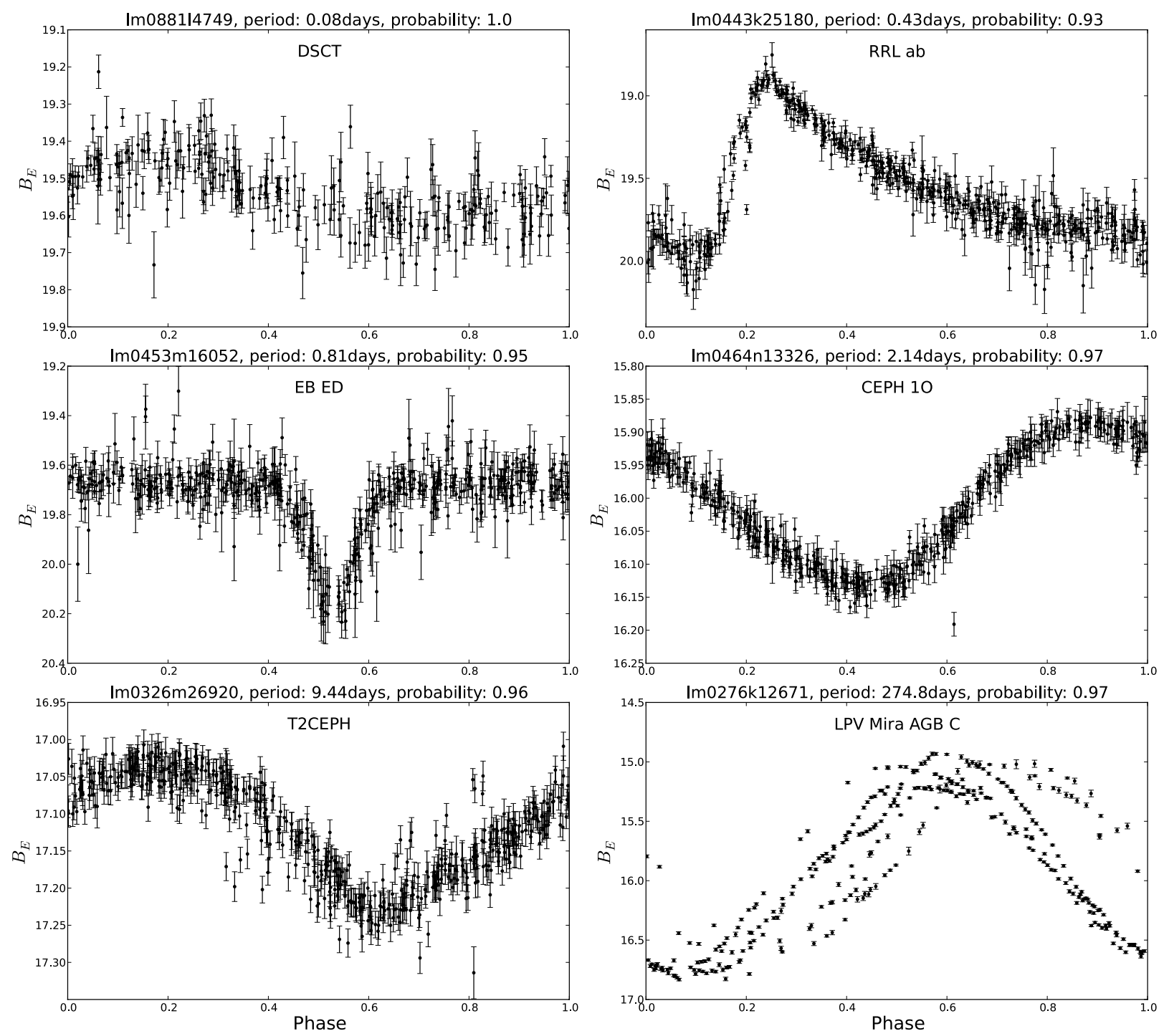

Fig. 13. Examples of phase-folded light curves of six new variable candidates.

see Appendix A for details of $R_{21}$. Each subclass is shown in a different color and is distinguishable from each other, which is a known property of RRL variables (Soszyński et al. 2009b). Figure 17 shows the period and $R_{21}$ of CEPH variable candidates, and each subclass is again relatively well distinguishable. Figure 18 displays the relation of period versus $\sigma$ of the LPVs.

\section{Summary}

We presented the first result of the EPOCH project: the classification of periodic variable stars in the EROS-2 LMC light-curve database. We first compiled the richest possible training set based mainly on the previously known OGLE variable stars. We chose 22 variability features based on the variable importance estimated by the random forest algorithm and then calculated the features using the visually examined training set. We then trained a random forest classification model using these variability features. We applied the model to the 29 million EROS-2 LMC sources and detected 117234 variable candidates. The catalog of the variable candidates containing EROS IDs, RA, Dec, colors (i.e., $B_{\mathrm{E}}-R_{\mathrm{E}}$ ), magnitudes (i.e., $B_{\mathrm{E}}$ ), periods, period $\mathrm{S} / \mathrm{N}$, probabilities and crossmatched OGLE/MACHO information is available at http://stardb.yonsei.ac.kr and at the CDS.
Note that the catalog contains all 150115 variable candidates without removal of the faint sources or low-period $\mathrm{S} / \mathrm{N}$ sources mentioned in Sect. 4.

The classification quality of any supervised machinelearning methods depends on the richness of the training set and informativeness of the features on which a classification model is trained. In this work, we used previously known OGLE variable sources to build a training set. Thus a classification model would not be feasible for selecting and classifying variable types that do not exist in the OGLE variable source catalogs. In future works, we will consider adding more variable sources of different types to increase the completeness of the training set. In addition, we visually removed sources without variability while building the training set, which might result in an incomplete training set because of unintended removal of weak-variability sources. Although we showed that the trained model was able to classify both strong and weak variability sources, additional investigation on an enhanced training set would be useful to increase classification quality. We used 22 variability features of the highest variable importance estimated with the random forest method. We did not see any noticeable improvements by using more or fewer features. Nevertheless, it would be interesting to perform a comprehensive feature selection based on a variety 
D.-W. Kim et al.: The EPOCH Project. I.
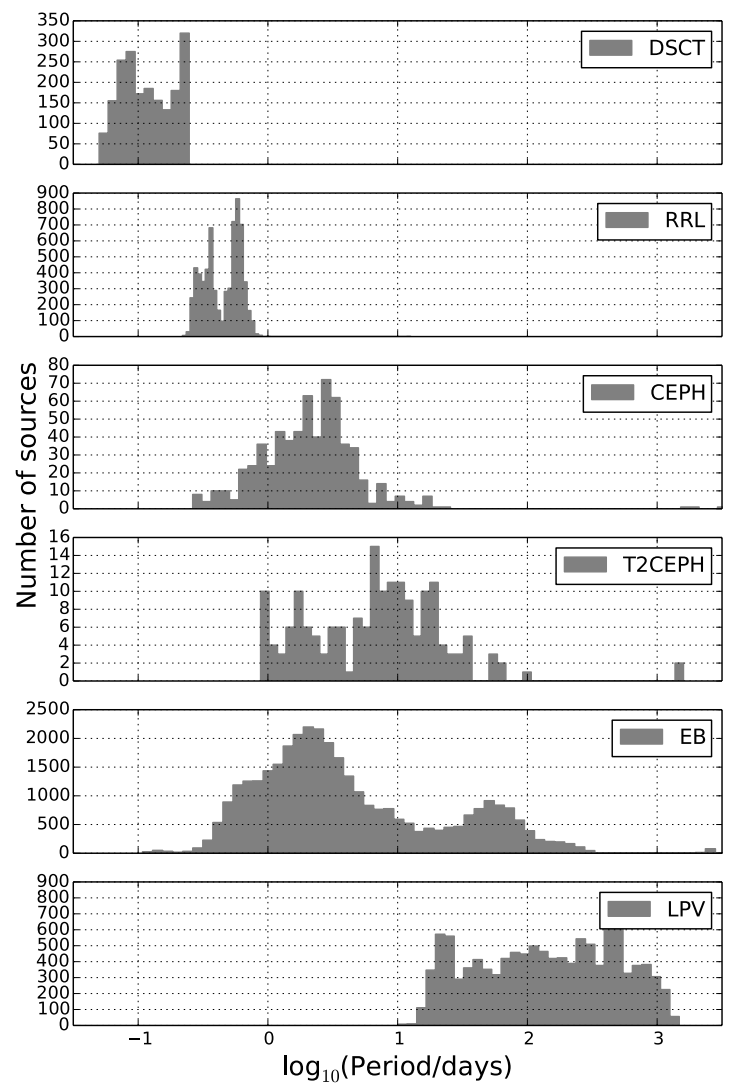

Fig. 14. Period histograms of the new variable candidates. From top to bottom: DSCTs, RRLs, CEPHs, T2CEPHs, EBs, and LPVs. Each type of variable star has a different period distribution.
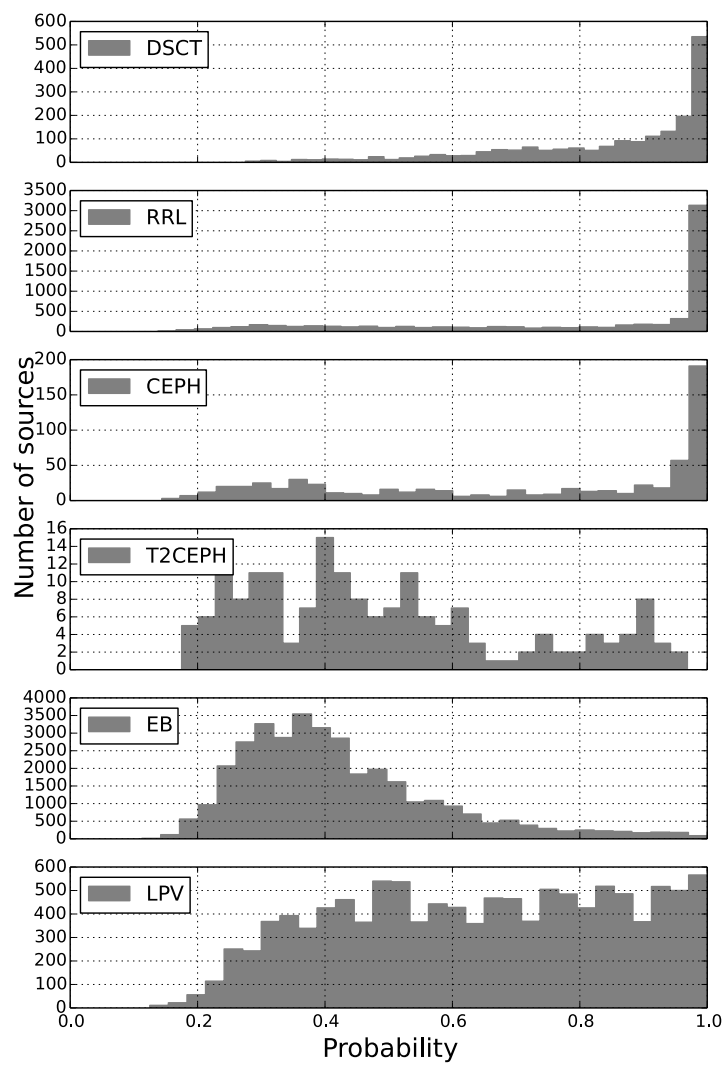

Fig. 15. Probability histograms of the new variable candidates. Although most of DSCTs, RRLs, and CEPHs show a probability higher than $90 \%$, a relatively large portion of candidates of T2CEPHs, EBs, and LPVs show a probability lower than 0.9 , which might be caused by misclassification within or between superclasses.

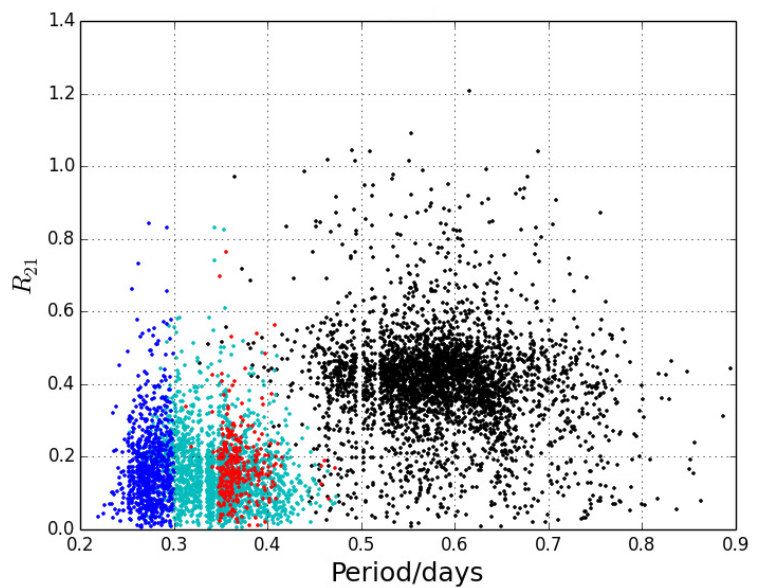

Fig. 16. Period versus $R_{21}$ for the new variable candidates of RRL type. RR ab: black circles, RR c: cyan circles, RR d: red circles and RR e: blue circles. The vertical blanks are attributed to spurious periods.

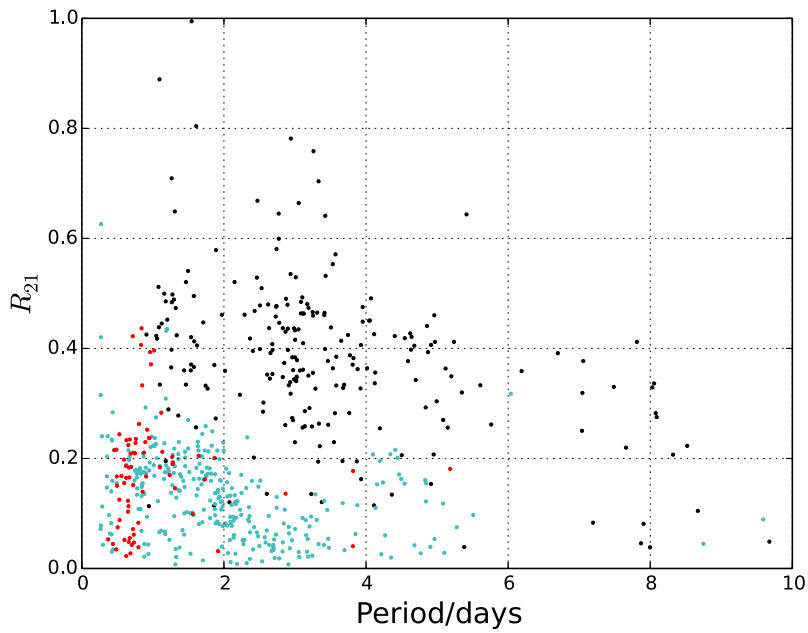

Fig. 17. Period versus $R_{21}$ for the new variable candidates of CEPH type. F: black circles, 1O: cyan circles, and others: red circles.
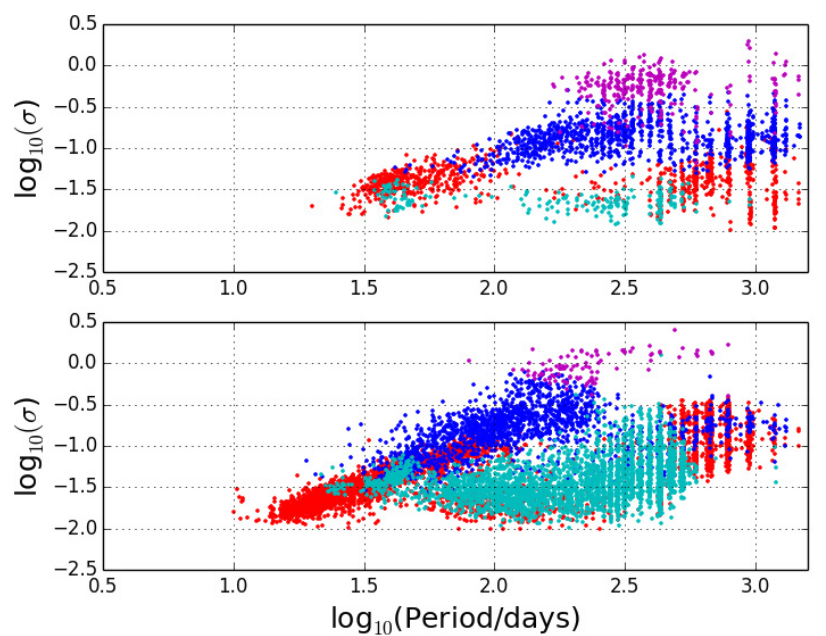

Fig. 18. Period versus $\sigma$ for the new variable candidates of LPV type. OSARG AGBs: red circles, OSARG RGBs: blue circles, SRV AGBs: cyan circles and Mira AGBs: magenta circles. The carbon-rich LPVs (top panel) show relatively longer periods than the oxygen-rich LPVs (bottom panel) (Soszyński et al. 2009a). Mira LPVs have larger amplitude than other LPVs, which is a known property of Mira variables (Soszyński et al. 2009a, 2013). The vertical lines at longer periods are caused by a sparse sampling of the periodogram derived by the LombScargle method. 
of methods (e.g. see Guyon \& Elisseeff 2003 and references therein) to find irrelevant and/or highly correlated features that could be removed without detriment to the classification quality.

In future works, we will apply a similar classification approach to the one presented for the EROS-2 SMC, Galactic bulge, and spiral arm databases to select and classify variable candidates.

Acknowledgements. The EROS-2 project was funded by the CEA and the CNRS through the IN2P3 and INSU institutes. J.B.M. acknowledges financial support from "Programme National de Physique Stellaire" (PNPS) of the CNRS/INSU, France. Work at Yonsei was supported by NRF grant 2012R1A1A2006924. The analysis in this paper has been performed using the Odyssey cluster supported by the FAS Research Computing Group at Harvard.

\section{Appendix A}

In this appendix, we explain each variability feature that we used to train the classification model. $Q_{3-1 \mid B-R}, \psi^{\mathrm{CS}}, \psi^{\eta}, \eta_{B-R}$, and $\eta^{e}$ have been developed for this work. Some other features were developed and/or used in other works such as Shin et al. (2009), Kim et al. (2011, 2012), Pichara et al. (2012) and Shin et al. (2012).

- Period and period S/N. In the top-left panel of Fig. A.1, we show the period versus period S/N. Most of the variable types are grouped in different regions. For instance, LPVs (magenta $\mathrm{x}$ ) have longer periods than others, such as RRLs, EBs, CEPHs, and DSCTs. The period was derived using the Lomb-Scargle algorithm (Lomb 1976) and is the highest peak in the periodogram.

We define the signal-to-noise ratio $(\mathrm{S} / \mathrm{N})$ of the highest peak as

$S / N=\frac{\max \left(p_{\mathrm{LS}}\right)-\bar{p}_{\mathrm{LS}}}{\sigma_{p_{\mathrm{LS}}}}$,

where $p_{\mathrm{LS}}$ is power of all frequencies in the periodogram, $\max$ is the maximum value, $\bar{p}_{\mathrm{LS}}$ is a mean power, and $\sigma_{p_{\mathrm{LS}}}$ is a standard deviation. Although the $\mathrm{S} / \mathrm{N}$ of a peak (Scargle 1982 ) is generally defined as

$S / N=\frac{\max \left(p_{\mathrm{LS}}\right)}{\bar{p}_{\mathrm{LS}}}$,

we used the alternative definition since we empirically found that $\sigma_{p_{\mathrm{LS}}}$ of peridograms of EROS-2 light curves are relatively large, which could be attributed to noise in the light curves such as systematic trends and high photometric uncertainty. The top-left panel of Fig. A.1 shows that the majority of non-variables (gray circles) and the field sources (contour line) have a lower period $\mathrm{S} / \mathrm{N}$ than other variables.

- $\eta . \eta$ measures the degree of trends (e.g. monotonic increase or decrease of flux in a long-term baseline) (von Neumann 1941). $\eta$ is defined as

$\eta=\frac{1}{(N-1) \sigma^{2}} \sum_{i=1}^{N-1}\left(m_{i+1}-m_{i}\right)^{2}$,

where $N$ is the total number of measurements of a light curve, $\sigma$ is the standard deviation, $m$ is the magnitude, and $i$ is the index of time. $\eta$ is known to be useful to separate light curves with long-term variation, as shown in the bottomright panel of Fig. A.1.
- Color and magnitude. Color and magnitude are powerful indices for variable separation because different variable types are generally placed at different regions in a color-magnitude diagram (CMD). The CMD of the training set is shown in the top-right panel of Fig. A.1. Although there is some overlap between classes, some classes are clearly distinguishable in the CMD, for example, LPVs.

- $Q_{3-1}$. $Q_{3-1}$ is the difference between the third quartile, $Q_{3}$, and the first quartile, $Q_{1}$, of a raw light curve. $Q_{1}$ is a split between the lowest $25 \%$ and the highest $75 \%$ of data. $Q_{3}$ is a split between the lowest $75 \%$ and the highest $25 \%$ of data.

- Cusum. Cusum is the range of a cumulative sum (e.g. see Ellaway 1978) of a light curve and is defined as

Cusum $=\max (S)-\min (S)$

$S_{l}=\frac{1}{N \sigma} \sum_{i=1}^{l}\left(m_{i}-\bar{m}\right)$,

where max (min) is the maximum (minimum) value of $S$ and $l=1,2, \ldots, N . \bar{m}$ is a mean magnitude. Cusum is generally high for light curves with long-term variability such as LPVs, BVs, and QSOs, while it is relatively low for light curves of short-periodic variables and non-variables (Kim et al. 2011).

- $Q_{3-1 \mid B-R}$ and $\psi^{\mathrm{CS}} \cdot Q_{3-1 \mid B-R}$ and $\psi^{\mathrm{CS}}$ are not the features derived from a raw light curve but the features derived from a $B_{\mathrm{E}}-R_{\mathrm{E}}$ light curve, $Q_{3-1 \mid B-R}$, and from a phase-folded light curve, $\psi^{\mathrm{CS}}$. We generated a $B_{\mathrm{E}}-R_{\mathrm{E}}$ light curve using only the simultaneous measurements from $B_{\mathrm{E}}$ and $R_{\mathrm{E}}$. If a variable star's flux variability is achromatic, the generated $B_{\mathrm{E}}-R_{\mathrm{E}}$ light curve follows the normal distribution (i.e., Gaussian noise). Otherwise it would show a certain variability in the $B_{\mathrm{E}}-R_{\mathrm{E}}$ light curve.

$\psi^{\mathrm{CS}}$ is the range of the cumulative sum (Cusum) of a phasefolded light curve. A phase-folded light curve is generated using the period estimated from the Lomb-Scargle method. For relatively short-period variables such as DSCTs, RRLs, CEPHs, and EBs, a phase-folded light curve would have a different shape from a raw light curve and thus embrace different variability characteristics. In the bottom-left panel of Fig. A.1, we show the scatter plot of $Q_{3-1 \mid B-R}$ versus $\psi^{\text {CS }}$ of short-period variables including DSCTs, RRLs, CEPHs, and EBs. The short-period variables are relatively well distinguishable. We do not show other types of variables because they are spread across the plane.

- $\psi^{\eta} . \psi^{\eta}$ is the $\eta$ index calculated from the phase-folded light curve. Dubath et al. (2011) used a conceptually identical feature with $\psi^{\eta}$, which they termed $P 2 p$ scatter.

- $\eta_{B-R}$. This is an $\eta$ index calculated from the $B_{\mathrm{E}}-R_{\mathrm{E}}$ light curve.

- $\eta^{e}$. Although $\eta$ is a powerful index for quantifying variability characteristics of a light curve, it does not take into account unequal sampling. Thus we define $\eta^{e}$ as

$$
\begin{aligned}
\eta^{e} & =\bar{w}\left(t_{N-1}-t_{1}\right)^{2} \frac{\sum_{i=1}^{N-1} w_{i}\left(m_{i+1}-m_{i}\right)^{2}}{\sigma^{2} \sum_{i=1}^{N-1} w_{i}} \\
w_{i} & =\frac{1}{\left(t_{i+1}-t_{i}\right)^{2}},
\end{aligned}
$$

where $t$ is the measurement epoch, $t_{i+1}>t_{i}$, and $\bar{w}$ is the mean value of $w_{i}$. The right panel in Fig. A.2 shows the $\eta^{e}$ histogram of RRLs, CEPHs, and EBs. Although the separation between the classes is not perfect, CEPHs are clearly distinguishable from others. We designed $\eta^{e}$ considering different sampling rates and baselines between light curves, 

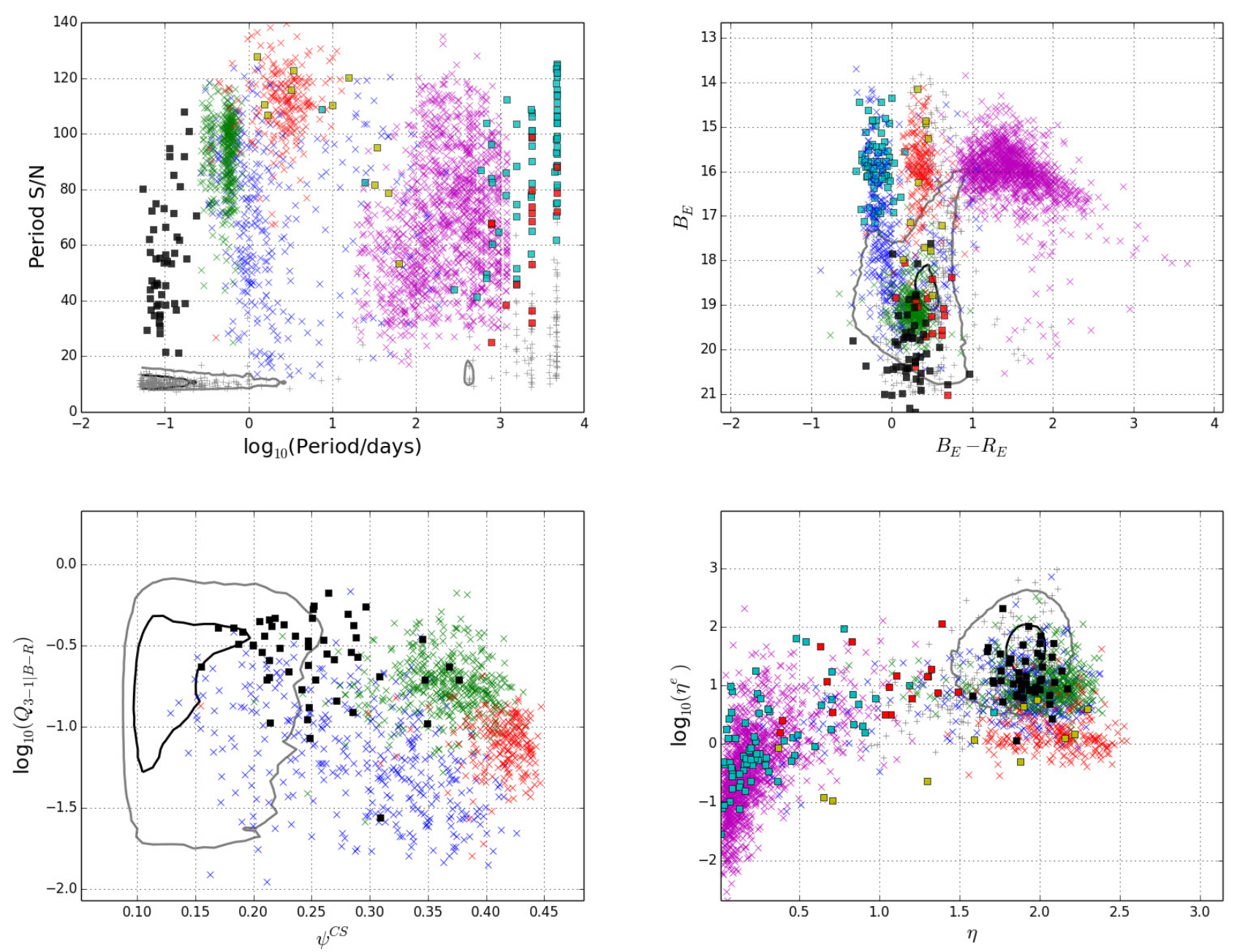

Fig. A.1. 2D scatter plots of variability features of the training set explained in Sect. 3.1. DSCTs: black squares, RRLs: green $\mathrm{x}$, CEPHs: red $\mathrm{x}$, EBs: blue x, T2CEPHs: yellow squares, LPVs: magenta x, QSOs: red squares, BVs: cyan squares, and NonVars: grey crosses. From left to right, clockwise: period versus period $\mathrm{S} / \mathrm{N}, B_{\mathrm{E}}-R_{\mathrm{E}}$ (i.e., color) versus $B_{\mathrm{E}}$ band magnitude, $\eta$ versus $\eta^{e}$, and $\psi^{\mathrm{CS}}$ versus $Q_{3-1 \mid B-R}$. The contour line shows spatial distribution of about 550k field sources. To generate the contour line, we built a $2 \mathrm{D}$ histogram of the field sources and then used the counts in each 2D-bin. The figure shows the two contour levels of 100 (thick gray line) and 1000 (thick black line). The majority of these field sources are probably non-variables. Different variable classes are separately grouped in the 2D space of variability features. We plot only one out of ten samples from the training set for better legibility of the figure. See text for details.
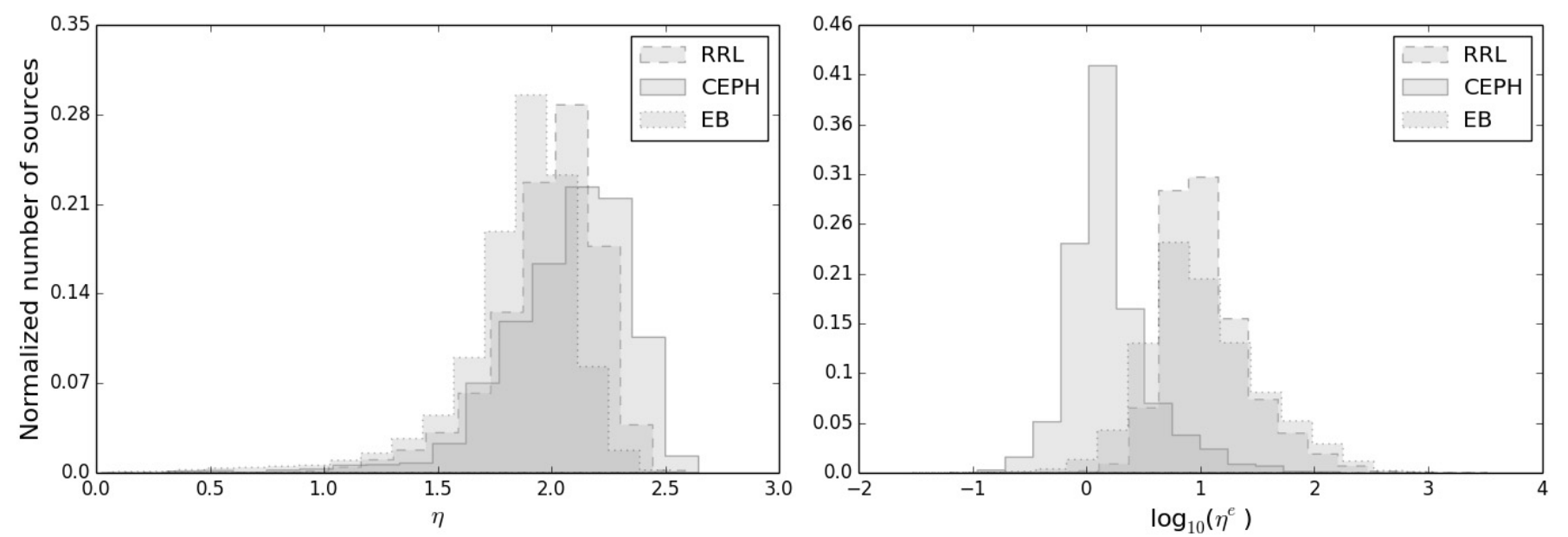

Fig. A.2. Histogram of $\eta$ (left panel) and $\eta^{e}$ (right panel) of RRLs (dashed), CEPHs (solid), and EBs (dotted). Using $\eta^{e}$, CEPHs are distinguishable from the other two classes. 
which is useful in some cases such as shown in Fig. A.2 and also in the bottom-right panel of Fig. A.1. Additional investigation, however, would be necessary to examine detailed characteristics of $\eta^{e}$.

- $\sigma, \gamma_{1}$ and $\gamma_{2} . \sigma$ is a standard deviation, $\gamma_{1}$ is a skewness and $\gamma_{2}$ is a kurtosis of a light curve. $\gamma_{1}$ and $\gamma_{2}$ are defined as

$$
\begin{aligned}
\gamma_{1}= & \frac{N}{(N-1)(N-2)} \sum_{i=1}^{N}\left(\frac{m_{i}-\bar{m}}{\sigma}\right)^{3} \\
\gamma_{2}= & \frac{N(N+1)}{(N-1)(N-2)(N-3)} \sum_{i=1}^{N}\left(\frac{m_{i}-\bar{m}}{\sigma}\right)^{4} \\
& -\frac{3(N-1)^{2}}{(N-2)(N-3)},
\end{aligned}
$$

- $J$ and $K . J$ is a Stetson $J$ index (Stetson 1996), which is calculated based on simultaneous measurements from light curves in two bands, $p$ and $q$, of the same star. It is defined as

$$
\begin{aligned}
& J=\frac{1}{N} \sum_{i=1}^{N} \operatorname{sign}\left(P_{i}\right) \sqrt{\left|P_{i}\right|} \\
& P_{i}=\delta_{p}(i) \delta_{q}(i) \\
& \delta_{p}(i)=\sqrt{\frac{N}{N-1}} \frac{m_{p, i}-\bar{m}}{\sigma_{p, i}},
\end{aligned}
$$

where $N$ is the total number of measurements, $i$ is an index for each measurement, and $\operatorname{sign}\left(P_{i}\right)$ is the sign of $P_{i} . \sigma_{p, i}$ is the standard error of $i_{\text {th }}$ measurement of band $p$. In case of the EROS-2 database, $p$ and $q$ indicate $B_{\mathrm{E}}$ and $R_{\mathrm{E}}$ bands.
Stetson $K$ is calculated using a single band light curve as

$$
K=\frac{1}{\sqrt{N}} \frac{\sum_{i=1}^{N}|\delta(i)|}{\sqrt{\sum_{i=1}^{N} \delta(i)^{2}}},
$$

A pure sinusoid signal has $K=0.900$ and Gaussian distribution has $K=0.798$.

- $K_{\mathrm{AC}} . K_{\mathrm{AC}}$ is Stetson $K$ index calculated over the autocorrelation function of a light curve. This feature is useful to separate long- or non-periodic variables including QSOs from periodic variables (see Kim et al. 2011, for details).

- $R_{21}, R_{31}, \phi_{21}$, and $\phi_{31}$. These features are derived using the Fourier decomposition (Petersen 1986; Grison 1994). $R_{k 1}$ is an amplitude ratio and $\phi_{k 1}$ is a phase difference, which is defined as

$$
\begin{aligned}
& R_{k 1}^{2} \quad=\frac{A_{k}^{2}+B_{k}^{2}}{A_{1}^{2}+B_{1}^{2}} \\
& \phi_{k 1}=\phi_{k}-k \phi_{1}, \text { where } \phi_{k}=\tan ^{-1}\left(-\frac{B_{k}}{A_{k}}\right) .
\end{aligned}
$$

To calculate $A_{k}$ and $B_{k}$, we adopted the Grison model-fitting procedure (Grison 1994), which first constructs a zero-mean light curve and then fits a Fourier series, defined as

$\sum_{i=1}^{M}\left[A_{i} \cos \left(i \frac{2 \pi}{P} t\right)+B_{i} \sin \left(i \frac{2 \pi}{P} t\right)\right]$,

where $M$ is the number of series to fit, $P$ is the derived period from the Lomb-Scargle method, and $t$ is the time epoch. The features $R_{k 1}$ and $\phi_{k 1}$ can distinguish different shapes of light curves and thus are useful to separate subclasses of shortperiod variables such as CEPHs (Beaulieu et al. 1995).

\section{Appendix B: Confusion matrix}

Table B.1. Performance of the classification model without considering subclasses.

\begin{tabular}{lrrrrrrrrrr}
\hline \hline Class & DSCT & RRL & CEPH & T2CEPH & EB & LPV & BV & QSO & NoneVar & Recall $(\%)$ \\
\hline DSCT & 253 & 1 & 0 & 0 & 0 & 0 & 0 & 0 & 1 & 99.2 \\
RRL & 3 & 2088 & 4 & 2 & 16 & 0 & 0 & 0 & 0 & 98.8 \\
CEPH & 0 & 10 & 1094 & 3 & 12 & 0 & 1 & 0 & 1 & 97.6 \\
T2CEPH & 0 & 0 & 8 & 37 & 10 & 1 & 0 & 0 & 0 & 66.1 \\
EB & 1 & 11 & 6 & 0 & 1652 & 20 & 0 & 0 & 14 & 96.9 \\
LPV & 0 & 0 & 0 & 1 & 6 & 6642 & 1 & 1 & 12 & 99.7 \\
BV & 0 & 0 & 0 & 0 & 2 & 0 & 353 & 1 & 6 & 97.5 \\
QSO & 0 & 0 & 0 & 0 & 0 & 1 & 2 & 71 & 7 & 87.7 \\
NoneVar & 2 & 0 & 0 & 0 & 6 & 12 & 5 & 4 & 1812 & 98.4 \\
Precision (\%) & 97.7 & 99.0 & 98.4 & 86.0 & 96.9 & 99.5 & 97.5 & 92.2 & 97.8 & $98.6,98.6$ \\
\hline
\end{tabular}


D.-W. Kim et al.: The EPOCH Project. I.

Table B.2. Performance of the classification model considering subclasses.

\begin{tabular}{lllllll}
\hline \hline Class & BV & CEPH_1O & CEPH_F & CEPH_Other & DSCT & EB_EC \\
\hline BV & 349 & 0 & 0 & 0 & 0 & 0 \\
CEPH_1O & 0 & 380 & 5 & 16 & 1 & 0 \\
CEPH_F & 0 & 10 & 608 & 3 & 0 & 0 \\
CEPH_Other & 0 & 19 & 2 & 57 & 0 & 0 \\
DSCT & 0 & 0 & 0 & 0 & 249 & 2 \\
EB_EC & 0 & 3 & 0 & 0 & 0 & 110 \\
EB_ED & 0 & 0 & 0 & 0 & 0 & 3 \\
EB_ED_ESD & 0 & 0 & 0 & 0 & 0 & 0 \\
EB_ESD & 1 & 0 & 1 & 0 & 0 & 24 \\
EB_Other & 0 & 0 & 1 & 0 & 0 & 9 \\
LPV_Mira_AGB_C & 0 & 0 & 0 & 0 & 0 & 0 \\
LPV_Mira_AGB_O & 0 & 0 & 0 & 0 & 0 & 0 \\
LPV_OSARG_AGB_C & 0 & 0 & 0 & 0 & 0 & 0 \\
LPV_OSARG_AGB_O & 0 & 0 & 0 & 0 & 0 & 0 \\
LPV_OSARG_RGB_C & 0 & 0 & 0 & 0 & 0 & 0 \\
LPV_OSARG_RGB_O & 2 & 0 & 0 & 0 & 0 & 4 \\
LPV_SRV_AGB_C & 0 & 0 & 0 & 0 & 0 & 0 \\
LPV_SRV_AGB_O & 1 & 0 & 0 & 0 & 0 & 1 \\
NoneVar & 6 & 0 & 0 & 0 & 2 & 0 \\
QSO & 0 & 0 & 0 & 0 & 0 & 0 \\
RRL_RRab & 0 & 0 & 0 & 2 & 0 & 1 \\
RRL_RRc & 0 & 0 & 0 & 0 & 0 & 2 \\
RRL_RRd & 0 & 1 & 0 & 0 & 0 & 0 \\
RRL_RRe & 0 & 0 & 0 & 0 & 2 & 0 \\
T2CEPH & 0 & 2 & 5 & 0 & 0 & 1 \\
Precision (\%) & 97.2 & 91.6 & 97.7 & 73.1 & 98.0 & 70.1 \\
\hline
\end{tabular}

Table B.2. continued.

\begin{tabular}{lllllll}
\hline \hline Class & EB_ED & EB_ED_ESD & EB_ESD & EB_Other & LPV_Mira_AGB_C & LPV_Mira_AGB_O \\
\hline BV & 0 & 0 & 1 & 1 & 0 & 0 \\
CEPH_1O & 0 & 0 & 6 & 0 & 0 & 0 \\
CEPH_F & 0 & 0 & 3 & 0 & 0 & 0 \\
CEPH_Other & 0 & 0 & 0 & 0 & 0 & 0 \\
DSCT & 0 & 0 & 0 & 0 & 0 & 0 \\
EB_EC & 2 & 0 & 77 & 6 & 0 & 0 \\
EB_ED & 708 & 3 & 73 & 1 & 0 & 0 \\
EB_ED_ESD & 52 & 4 & 15 & 0 & 0 & 0 \\
EB_ESD & 106 & 1 & 405 & 2 & 0 & 0 \\
EB_Other & 16 & 0 & 30 & 8 & 0 & 6 \\
LPV_Mira_AGB_C & 0 & 0 & 0 & 0 & 250 & 133 \\
LPV_Mira_AGB_O & 0 & 0 & 0 & 0 & 3 & 0 \\
LPV_OSARG_AGB_C & 0 & 0 & 0 & 0 & 0 & 0 \\
LPV_OSARG_AGB_O & 0 & 0 & 2 & 0 & 1 & 0 \\
LPV_OSARG_RGB_C & 0 & 0 & 0 & 0 & 0 & 0 \\
LPV_OSARG_RGB_O & 0 & 0 & 0 & 0 & 0 & 14 \\
LPV_SRV_AGB_C & 0 & 0 & 0 & 0 & 29 & 0 \\
LPV_SRV_AGB_O & 0 & 0 & 0 & 0 & 5 & 0 \\
NoneVar & 0 & 0 & 2 & 0 & 0 & 0 \\
QSO & 0 & 0 & 0 & 0 & 1 & 0 \\
RRL_RRab & 1 & 0 & 1 & 0 & 0 & 0 \\
RRL_RRc & 0 & 0 & 2 & 0 & 0 & 0 \\
RRL_RRd & 0 & 0 & 2 & 0 & 0 & 06.9 \\
RRL_RRe & 0 & 0 & 0 & 0 & 0 & 0 \\
T2CEPH & 1 & 0 & 4 & 0 & 06.5 & \\
Precision $(\%)$ & 79.9 & 50.0 & 65.0 & 44.4 & 0 & 0 \\
\hline
\end{tabular}


Table B.2. continued.

\begin{tabular}{|c|c|c|c|c|c|c|}
\hline$\overline{\text { Class }}$ & $\begin{array}{l}\text { LPV_OSARG_ } \\
\text { AGB_C }\end{array}$ & $\begin{array}{l}\text { LPV_OSARG_ } \\
\text { AGB_O }\end{array}$ & $\begin{array}{l}\text { LPV_OSARG_ } \\
\text { RGB_C }\end{array}$ & $\begin{array}{l}\text { LPV_OSARG_ } \\
\text { RGB_O }\end{array}$ & $\begin{array}{l}\text { LPV_SRV } \\
\text { AGB_C }\end{array}$ & $\begin{array}{l}\text { LPV_SRV__ } \\
\text { AGB_O }\end{array}$ \\
\hline $\mathrm{BV}$ & 0 & 0 & 0 & 0 & 0 & 0 \\
\hline CEPH_1O & 0 & 0 & 0 & 0 & 0 & 0 \\
\hline CEPH_F & 0 & 0 & 0 & 0 & 0 & 0 \\
\hline CEPH_Other & 0 & 0 & 0 & 0 & 0 & 0 \\
\hline $\mathrm{DSCT}^{-}$ & 0 & 0 & 0 & 0 & 0 & 0 \\
\hline EB_EC & 0 & 0 & 0 & 3 & 0 & 0 \\
\hline EB_ED & 1 & 0 & 0 & 1 & 0 & 0 \\
\hline EB_ED_ESD & 0 & 0 & 0 & 0 & 0 & 0 \\
\hline EB_ESD & 0 & 0 & 0 & 5 & 0 & 0 \\
\hline EB_Other & 0 & 1 & 0 & 4 & 0 & 0 \\
\hline LPV_Mira_AGB_C & 0 & 1 & 0 & 3 & 18 & 5 \\
\hline LPV_Mira_AGB_O & 0 & 0 & 0 & 0 & 2 & 24 \\
\hline LPV_OSARG_AGB_C & 323 & 123 & 0 & 15 & 95 & 7 \\
\hline LPV_OSARG_AGB_O & 66 & 1288 & 1 & 62 & 15 & 153 \\
\hline LPV_OSARG_RGB_C & 2 & 3 & 3 & 9 & 3 & 2 \\
\hline LPV_OSARG_RGB_O & 2 & 82 & 2 & 797 & 12 & 42 \\
\hline LPV_SRV_AGB_C ${ }^{-}$ & 40 & 29 & 0 & 10 & 1105 & 39 \\
\hline LPV_SRV_AGB_O & 7 & 163 & 3 & 45 & 45 & 1550 \\
\hline NoneVar & 8 & 3 & 1 & 2 & 1 & 1 \\
\hline QSO & 0 & 0 & 0 & 0 & 0 & 0 \\
\hline RRL_RRab & 0 & 0 & 0 & 0 & 0 & 0 \\
\hline RRL_RRc & 0 & 0 & 0 & 0 & 0 & 0 \\
\hline RRL_RRd & 0 & 0 & 0 & 0 & 0 & 0 \\
\hline RRL_RRe & 0 & 0 & 0 & 0 & 0 & 0 \\
\hline T2CEPH & 0 & 0 & 0 & 0 & 0 & 2 \\
\hline Precision (\%) & 71.9 & 76.1 & 30.0 & 83.4 & 85.3 & 84.9 \\
\hline
\end{tabular}

Table B.2. continued.

\begin{tabular}{lllllllll}
\hline \hline Class & NoneVar & QSO & RRL_RRab & RRL_RRc & RRL_RRd & RRL_RRe & T2CEPH & Recall $(\%)$ \\
\hline BV & 11 & 1 & 0 & 0 & 0 & 0 & 0 & 96.1 \\
CEPH_1O & 1 & 0 & 1 & 5 & 1 & 1 & 0 & 91.1 \\
CEPH_F & 0 & 0 & 0 & 0 & 0 & 0 & 1 & 97.3 \\
CEPH_Other & 0 & 0 & 0 & 1 & 1 & 0 & 0 & 71.3 \\
DSCT & 2 & 0 & 0 & 0 & 0 & 2 & 0 & 97.6 \\
EB_EC & 0 & 0 & 3 & 2 & 0 & 0 & 3 & 52.6 \\
EB_ED & 6 & 0 & 0 & 0 & 0 & 0 & 0 & 88.9 \\
EB_ED_ESD & 0 & 0 & 0 & 0 & 0 & 0 & 0 & 5.6 \\
EB_ESD & 1 & 0 & 9 & 0 & 0 & 0 & 0 & 73.0 \\
EB_Other & 4 & 0 & 0 & 0 & 0 & 0 & 0 & 11.0 \\
LPV_Mira_AGB_C & 0 & 0 & 0 & 0 & 0 & 0 & 0 & 88.3 \\
LPV_Mira_AGB_O & 0 & 0 & 0 & 0 & 0 & 0 & 0 & 82.1 \\
LPV_OSARG_AGB_C & 4 & 0 & 0 & 0 & 0 & 0 & 0 & 57.0 \\
LPV_OSARG_AGB_O & 6 & 0 & 0 & 0 & 0 & 0 & 0 & 80.8 \\
LPV_OSARG_RGB_C & 1 & 0 & 0 & 0 & 0 & 0 & 0 & 13.0 \\
LPV_OSARG_RGB_O & 1 & 0 & 0 & 0 & 0 & 0 & 0 & 84.4 \\
LPV_SRV_AGB_C & 1 & 0 & 0 & 0 & 0 & 0 & 0 & 88.2 \\
LPV_SRV_AGB_O & 1 & 0 & 0 & 0 & 0 & 0 & 1 & 84.4 \\
NoneVar & 1807 & 6 & 0 & 0 & 0 & 1 & 0 & 98.2 \\
QSO & 5 & 74 & 0 & 0 & 0 & 0 & 0 & 92.5 \\
RRL_RRab & 0 & 0 & 1632 & 1 & 0 & 0 & 0 & 99.6 \\
RRL_RRc & 0 & 0 & 1 & 306 & 2 & 6 & 0 & 95.9 \\
RRL_RRd & 0 & 0 & 2 & 25 & 56 & 0 & 0 & 65.1 \\
RRL_RRe & 0 & 0 & 0 & 10 & 0 & 59 & 0 & 83.1 \\
T2CEPH & 1 & 0 & 0 & 0 & 0 & 0 & 40 & 71.4 \\
Precision (\%) & 97.6 & 91.4 & 99.0 & 87.4 & 93.3 & 85.5 & 88.9 & $87.7,86.3$ \\
\hline
\end{tabular}


D.-W. Kim et al.: The EPOCH Project. I.

\section{References}

Alard, C., \& Lupton, R. H. 1998, ApJ, 503, 325

Alcock, C. 2000, ApJ, 542, 281

Alcock, C. 2001, Variable Stars in the Large Magellanic Clouds, VizieR Online Data Catalog, II/247

Alcock, C., Allsman, R. A., Axelrod, T. S., et al. 1996, AJ, 111, 1146

Ansari, R. 1996, Vistas in Astron., 40, 519

Ansari, R., \& EROS Collaboration 2001, in Gravitational Lensing: Recent Progress and Future Goals, eds. T. G. Brainerd, \& C. S. Kochanek, ASP Conf. Ser., 237, 235

Beaulieu, J. P., Grison, P., Tobin, W., et al. 1995, A\&A, 303, 137

Beaulieu, J.-P., de Wit, W. J., Lamers, H. J. G. L. M., et al. 2001, A\&A, 380, 168

Breiman, L. 1996, Machine Learning, 24, 123

Breiman, L. 2001, Machine Learning, 45, 5

Carliles, S., Budavári, T., Heinis, S., Priebe, C., \& Szalay, A. S. 2010, ApJ, 712, 511

Carretta, E., Gratton, R. G., Clementini, G., \& Fusi Pecci, F. 2000, ApJ, 533, 215

Catelan, M. 2009, Ap\&SS, 320, 261

Dubath, P., Rimoldini, L., Süveges, M., et al. 2011, MNRAS, 414, 2602

Dubath, P., Lecoeur, I., Rimoldini, L., et al. 2012, in IAU Symp. 285, eds.

E. Griffin, R. Hanisch, \& R. Seaman, 309

Ellaway, P. 1978, Electroenceph. Clin. Neurophysiol., 45, 302

Feast, M. W., \& Catchpole, R. M. 1997, MNRAS, 286, L1

Feast, M. W., Glass, I. S., Whitelock, P. A., \& Catchpole, R. M. 1989, MNRAS, 241,375

Freedman, W. L., Madore, B. F., Gibson, B. K., et al. 2001, ApJ, 553, 47

Graczyk, D., Soszyński, I., Poleski, R., et al. 2011, Acta Astron., 61, 103

Grison, P. 1994, A\&A, 289, 404

Guyon, I., \& Elisseeff, A. 2003, J. Mach. Learn. Res., 3, 1157

Haschke, R., Grebel, E. K., \& Duffau, S. 2011, AJ, 141, 158

Hubble, E., \& Humason, M. L. 1931, ApJ, 74, 43

Keller, S. C., Bessell, M. S., Cook, K. H., Geha, M., \& Syphers, D. 2002, AJ, 124,2039
Kim, D.-W., Protopapas, P., Byun, Y.-I., et al. 2011, ApJ, 735, 68

Kim, D.-W., Protopapas, P., Trichas, M., et al. 2012, ApJ, 747, 107

Knapp, G. R., Pourbaix, D., Platais, I., \& Jorissen, A. 2003, A\&A, 403, 993

Kozlowski, S., Onken, C. A., Kochanek, C. S., et al. 2013, ApJ, 775, 92

Lemaître, G. 1927, Ann. Soc. Sci. Bruxelles, 47, 49

Lomb, N. R. 1976, Ap\&SS, 39, 447

Marquette, J. B., Beaulieu, J. P., Buchler, J. R., et al. 2009, A\&A, 495, 249

Oort, J. H., \& Plaut, L. 1975, A\&A, 41, 71

Petersen, J. O. 1986, A\&A, 170, 59

Pichara, K., Protopapas, P., Kim, D.-W., Marquette, J.-B., \& Tisserand, P. 2012, MNRAS, 427, 1284

Poleski, R., Soszyński, I., Udalski, A., et al. 2010, Acta Astron., 60, 1

Quinlan, J. R. 1993, C4.5: programs for machine learning (San Francisco, CA, USA: Morgan Kaufmann Publishers Inc.)

Richards, J. W., Starr, D. L., Butler, N. R., et al. 2011, ApJ, 733, 10

Riess, A. G., Macri, L., Casertano, S., et al. 2011, ApJ, 730, 119

Scargle, J. D. 1982, ApJ, 263, 835

Shin, M., Sekora, M., \& Byun, Y. 2009, MNRAS, 400, 1897

Shin, M.-S., Yi, H., Kim, D.-W., Chang, S.-W., \& Byun, Y.-I. 2012, AJ, 143, 65

Soszyński, I., Poleski, R., Udalski, A., et al. 2008a, Acta Astron., 58, 163

Soszyński, I., Udalski, A., Szymański, M. K., et al. 2008b, Acta Astron., 58, 293

Soszyński, I., Udalski, A., Szymañski, M. K., et al. 2009a, Acta Astron., 59, 239

Soszyński, I., Udalski, A., Szymański, M. K., et al. 2009b, Acta Astron., 59, 1

Soszyński, I., Udalski, A., Szymański, M. K., et al. 2013, Acta Astron., 63, 21

Spano, M., Mowlavi, N., Eyer, L., et al. 2011, A\&A, 536, A60

Stetson, P. B. 1996, PASP, 108, 851

Storm, J., Gieren, W., Fouqué, P., et al. 2011, A\&A, 534, A94

Tisserand, P., Marquette, J. B., Beaulieu, J. P., et al. 2004, A\&A, 424, 245

Tisserand, P., Le Guillou, L., Afonso, C., et al. 2007, A\&A, 469, 387

Tisserand, P., Wood, P. R., Marquette, J. B., et al. 2009, A\&A, 501, 985

Udalski, A., Kubiak, M., \& Szymanski, M. 1997, Acta Astron., 47, 319

Vivas, A. K., Zinn, R., Andrews, P., et al. 2001, ApJ, 554, L33

von Neumann, J. 1941, Ann. Math. Statist., 12, 367 